\title{
Morphological influences on the recognition of monosyllabic monomorphemic words
}

\author{
R.H. Baayen ${ }^{\text {a,* }}$, L.B. Feldman ${ }^{\mathrm{b}}$, R. Schreuder ${ }^{\mathrm{c}}$ \\ ${ }^{\text {a }}$ Radboud University Nijmegen and Max Planck Institute for Psycholinguistics, P.O. Box 310, 6500 AH Nijmegen, The Netherlands \\ ${ }^{\mathrm{b}}$ State University of New York at Albany, Department of Psychology, SS112 Albany, NY 12222, USA \\ ${ }^{\mathrm{c}}$ Radboud University Nijmegen, P.O. Box 310, 6500 AH Nijmegen, The Netherlands
}

Received 15 July 2005; revision received 28 March 2006

\begin{abstract}
Balota et al. [Balota, D., Cortese, M., Sergent-Marshall, S., Spieler, D., \& Yap, M. (2004). Visual word recognition for single-syllable words. Journal of Experimental Psychology: General, 133, 283-316] studied lexical processing in word naming and lexical decision using hierarchical multiple regression techniques for a large data set of monosyllabic, morphologically simple words. The present study supplements their work by making use of more flexible regression techniques that are better suited for dealing with collinearity and non-linearity, and by documenting the contributions of several variables that they did not take into account. In particular, we included measures of morphological connectivity, as well as a new frequency count, the frequency of a word in speech rather than in writing. The morphological measures emerged as strong predictors in visual lexical decision, but not in naming, providing evidence for the importance of morphological connectivity even for the recognition of morphologically simple words. Spoken frequency was predictive not only for naming but also for visual lexical decision. In addition, it co-determined subjective frequency estimates and norms for age of acquisition. Finally, we show that frequency predominantly reflects conceptual familiarity rather than familiarity with a word's form.
\end{abstract}

(C) 2006 Elsevier Inc. All rights reserved.

Keywords: Lexical processing; Morphology; Regression; Mental lexicon; Lexical connectivity

Balota, Cortese, Sergent-Marshall, Spieler, and Yap (2004) have cautioned researchers in the field about the drawbacks of factorial designs where variables are manipulated in a noncontinuous manner and effects are assessed in terms of the presence or absence of a significant effect. They have eloquently demonstrated for us the power of regression analyses based on hundreds or

\footnotetext{
* Corresponding author. Fax: +31 243521213.

E-mail addresses: baayen@mpi.nl (R.H. Baayen), 1f503@ albany.edu (L.B. Feldman), schreude@mpi.nl (R. Schreuder).
}

even thousands of data points and the potential to consider simultaneously, as predictors, many properties of words that historically have been factorially manipulated in separate experiments. Moreover, they have demonstrated that because regression analyses give us the potential to examine the proportion of variance that a set of variables can account for, they permit us to make comparisons across experimental tasks (e.g., lexical decision and naming) or across subject groups (e.g., older and younger readers).

According to Balota et al., "The search for a significant effect does not typically motivate researchers to 
report the amount of variance that a given factor accounts for in a design. The latter information may ultimately be more important than the effects that reach the magical level of significance" (p. 285). From their regression analyses, multiple insights based on a new type of evidence emerge. One insight is that the naming task is relatively more influenced by form variables and that the lexical decision task is more influenced by semantic variables. A second is that frequency plays a more substantial role in the lexical decision task than in the naming task.

In their rich and influential paper, Balota and colleagues have tackled longstanding controversies about the role of neighborhood density and have compared several of the well-published measures of frequency (Baayen, Piepenbrock, \& Gulikers, 1995, CELEX; Kučera \& Francis, 1967; MetaMetrics, 2003; Zeno, Ivens, Millard, \& Duvvuri, 1995; HAL (1997)) with respect to their linear (and quadratic) contributions to performance in the lexical decision and naming tasks. They determined that the Zeno frequency norms (Zeno et al., 1995) had a "consistently large influence across groups and tasks." Therefore, in subsequent multiple regression analyses, justified by the percentage of variance it accounted for, they focused on the (linear) contributions of Zeno frequency norms as well as the contribution of subjective frequency (Balota, Pilotti, \& Cortese, 2001) to performance in visual lexical decision and word naming.

In the primary regression analyses based on a largescale data set, they made use of a hierarchical regression analysis where they treated their expansive collection of variables in three steps. In step 1, they included a set of indicator variables for the phonetic features of the word's initial segment, in order to capture variation caused by the differences in sensitivity of the voice key to the acoustic properties of the speech signal. Lexical predictors including word length, neighborhood size, various consistency measures as well as subjective frequency (Balota et al., 2001) and objective frequency based on Zeno et al. (1995) were entered into the model at step 2 . Then, in step 3, they brought semantic variables into the regression analyses, dividing them into three blocks. The first block included Nelson set size (Nelson, McEvoy, \& Schreiber, 1998), and imageability based on Toglia and Battig (1978). The second block included imageability based on Cortese and Fugett (2004) and the third block included semantic connectivity measures from Steyvers and Tanenbaum (2005).

The study of Balota et al. represents a rigorous analysis that is consistent with 20 years of research on word recognition. Nevertheless, Balota et al. also raised a number of methodological issues inviting further research and clarification. A first such issue is potential non-linearities in the relation between predictors and response latencies. Balota and colleagues observed a non-linear relation between frequency and lexical deci- sion latencies in a univariate regression, but did not explore this non-linearity further in multiple regression. The exclusion of relevant non-linear terms from multiple regression can induce otherwise unnecessary interactions, however.

A second issue concerns the order in which variables are entered into the hierarchical regression model. The advantage of hierarchical regression is that one can investigate the explanatory value of a block of variables over and above the explanatory value of the variables entered in preceding blocks. By implication, in hierarchical regression the researcher must decide where to allocate each variable. For instance, Balota et al. assigned word frequency to the second block. While this decision allowed them to ascertain the predictivity of the semantic variables entered in the third block over and above frequency, the consequence is that the specific contribution of frequency itself over and above the other variables in the model remains unspecified. For example (see Balota et al., 2004, Table 5), we learn that collectively the lexical variables had an $R^{2}$ of 0.42 for younger readers in the lexical decision task but we cannot isolate the unique contribution of objective frequency based on the Zeno count. This falls short of the goal "to establish the unique variance that a given predictor accounts for" (p. 285).

Balota et al. (2004) considered frequency measures along with the lexical rather than the semantic predictors. However, when compared with variables such as word length and neighborhood size, frequency has a decidedly semantic nature. For one, words but not nonwords possess a frequency. In addition, high frequency words tend to score higher on indices of semantic richness including number of associations (see, e.g., Balota et al., 2004, Table 4) and morphologically simple words that are high in frequency tend to have more morphological relatives than do lower frequency words (Schreuder \& Baayen, 1997). Perhaps most compelling from a non-morphological perspective is that an examination of Table 4 of Balota and his colleagues reveals stronger simple correlations of word frequency with semantic than with lexical variables. For example, objective frequency correlates with connectivity according to Steyvers and Tanenbaum (2005), WordNet connectivity, and Toglia and Battig imageability with $R^{2}$ values of $0.59,0.46$, and 0.78 , respectively. By contrast, its highest correlation with a form variable (viz., neighborhood size) is 0.13 .

A more general question that permeates any serious investigation in the domain of lexical processing is how to deal with the tight correlational structure between almost all lexical variables. As collinearity in the data matrix may cause severe problems for regression analysis, it is worth considering what options are available for neutralizing its potentially harmful effects. The solution of Balota et al. was hierarchical regression. 
This is one option, but, as we shall see, there are alternatives that sometimes lead to slightly different conclusions. In the present study, we make use of an alternative approach that has the important advantage that it provides insight into the explanatory value of individual predictors (and obviates the need to assign predictors to individual blocks).

A third issue that was acknowledged by Balota et al. is that their list of variables is not all-inclusive. From a morphological perspective, any follow up to the Balota study would benefit from the inclusion of morphologically defined variables so as to permit further insights into the processes that underlie word recognition.

Three morphological variables that are gaining prominence in the experimental literature are morphological family size (Schreuder \& Baayen, 1997), its elaborations inflectional and derivational entropy (Moscoso del Prado Martín, Kostić, \& Baayen, 2004; Tabak, Schreuder, \& Baayen, 2005) as well as the ratio of noun-to-verb frequency based on the sums of all nominal and verbal inflected forms (Baayen \& Moscoso del Prado Martín, 2005; Feldman \& Basnight-Brown, 2005; Tabak et al., 2005). All these variables are relevant for the processing of not only complex but also morphologically simple words. None were considered in the study of Balota et al. (2004).

A word's morphological family size is the number of complex word types in which a word occurs as a constituent. This measure of a word's morphological connectivity in the mental lexicon has been found to correlate negatively with visual lexical decision latencies (Bertram, Baayen, \& Schreuder, 2000; Schreuder \& Baayen, 1997), and with the magnitude of morphological facilitation in a priming task (Feldman \& Pastizzo, 2003). Words with greater morphological connectivity tend to be processed faster than words with little morphological connectivity.

The accumulated evidence suggests that the effect of morphological family size is semantic in nature. Removal of opaque family members from the family size counts has been observed to lead to improved correlations of family size and decision latency (Schreuder \& Baayen, 1997). For inflected words with stem changes, such as the Dutch past particple ge-vocht-en (from the verb vecht-en, 'to fight'), the family size of the verb is the appropriate predictor, and not the family size of the spuriously embedded noun vecht (De Jong, Schreuder, \& Baayen, 2003). Homonymic roots in Hebrew, and interlingual homographs bear further witness to the semantic locus of the family size effect. When a Hebrew word such as meraggel 'spy' is read, the count of family members in the same semantic field correlates negatively with lexical decision latencies, while the count of family members in other semantic fields (e.g., regel, 'foot') reveals a positive correlation (Moscoso del Prado Martín et al., 2005). Similarly, for Dutch-English interlingual homographs in Dutch or English lexical decision, both the Dutch and the English morphological family size counts are predictive, with opposite sign, depending on the language of the filler materials. If the filler materials are Dutch, there is facilitation from the Dutch and inhibition from the English family members. If the filler materials are English, the effect reverses. In generalized lexical decision, in which subjects are asked whether a letter string is a word in either English or Dutch, the two family size counts are both facilitatory (Dijkstra, Moscoso del Prado Martín, Schulpen, Schreuder, \& Baayen, 2005). Further evidence favoring a semantic locus was obtained in a study addressing morphological families in Finnish, which tend to be an order of magnitude larger than Dutch or English families. A study of Finnish lexical processing in reading revealed that semantic coherence within subfamilies is crucial for a solid family size effect to emerge (Moscoso del Prado Martín, Bertram, Häikiö, Schreuder, \& Baayen, 2004).

The family size effect was introduced by Schreuder and Baayen (1997) in a study that observed significant effects of a factorial contrast in family size when the summed frequencies of the family members were held constant, whereas a factorial contrast in the cumulated token frequencies of the family members was not predictive when the family size type count was held constant. The importance of types rather than tokens was replicated subsequently by De Jong et al. (2003). This robust effect, however, is somewhat counterintuitive, in the sense that one would expect less well-known family members to have a reduced contribution to the family size effect. Recently, Moscoso del Prado Martín and Kostić et al. (2004) showed that this incongruity can be resolved by replacing the family size count by Shannon's entropy calculated for the probability distribution of the words in the morphological family. The derivational entropy measure, which weights the family members for their token frequencies, gauges the amount of information carried by a word's derivational paradigm. Moscoso del Prado Martín and Kostić et al. (2004) also developed a parallel entropy measure for a word's inflectional paradigm, the inflectional entropy, following up on earlier work by Kostić (1995) and Kostić et al. (2003). Whereas Moscoso del Prado Martín and Kostić et al. (2004) proposed a single measure (expressed in bits of information) incorporating the frequency effect, the morphological family size effect, and the inflectional family size effect, we have opted for gauging the explanatory potential of these variables separately, especially as recent studies addressing the distributional properties of regular and irregular verbs and the associated consequences for lexical processing revealed these separate measures to be significant independent predictors (Baayen \& Moscoso del Prado Martín, 2005; Tabak et al., 2005).

An important goal of the present study is, therefore, to extend the approach of Balota et al. to the domain of 
morphology, and to ascertain whether morphological variables are predictive for the processing of morphologically simple words in a large-scale regression study when a wide range of covariates are taken into account.

A second, more theoretical but as we shall see highly related goal is to enhance our understanding of what is measured by frequency of occurrence. On the one hand, the adequacy of pure frequency of occurrence has been challenged by studies on subjective familiarity (Balota et al., 2001; Gernsbacher, 1984; Shapiro, 1969) and age of acquisition (Barry, Hirsh, Johnston, \& Williams, 2001; Brysbaert, 1996; Brysbaert, Lange, \& Van Wijnendaele, 2000; Carroll \& White, 1973a, 1973b; Zevin \& Seidenberg, 2002). On the other hand, the interpretation of word frequency as a pure lexical form effect has been challenged by Balota and Chumbley (1984, but see Monsell, Doyle, and Haggard, 1989) and more recently by Bates et al. (2003) and, of course, Balota et al. (2004). We will show that the true complexity of the frequency variable has been underestimated, and we will argue that frequency primarily captures conceptual familiarity.

We address these issues by means of a reanalysis of the monosyllabic monomorphemic nouns and verbs studied by Balota et al. Along the way, we will provide some advancements with respect to the three methodological issues identified by Balota et al. as areas for future improvement.

\section{Materials}

From the Balota database, we selected those words that according to the CELEX lexical database are simplex nouns or simplex verbs. For words with conversion alternants, CELEX lists both forms, one form as simplex, and the other as a conversion alternant. For instance, work is listed both as a verb, (work) [V], and as a conversion noun, ((work) [V]) [N]. For such words, we followed CELEX when assigning word category. For work, this decision meant that it was classified as a simplex verb. However, we added the ratio of the frequencies of the nominal and verbal readings, henceforth the noun-toverb ratio, as a covariate in order to control for the relative probabilities when a word belongs to more than one word category. A second criterion for inclusion was that response latencies should be available in the Balota et al. database for both the lexical decision and the word naming measures.

We excluded some 30 words for which we did not have consistency measures (described below). In this way, we formed a list of 2284 words, 1452 nouns, and 832 verbs. For each of these words, we added the byitem visual lexical decision latencies and naming latencies of the young participants from the Balota database. We also included the by-item subjective frequency rat- ings from this database. To this list of 2284 words with three behavioral measures, we added 24 covariates.

\section{Variables of lexical form}

We considered 10 measures for phonological and orthographic consistency: the token and type counts of forward (spelling-to-sound) and backward (sound-tospelling) inconsistent words (enemies), the token and type counts of phonological and orthographic neighbors, and the token and type counts of the friends (the consistent words).

In addition to these measures for orthographic consistency, we included as measures of orthographic form the length of the word (in letters), its number of neighbors or neighborhood density (Coltheart, Davelaar, Jonasson, \& Besner, 1977), and its mean logarithmic bigram token frequency, calculated over all words and all positions in these words.

We also included indicator variables to control for the differential sensitivity of the voice key to the acoustic properties of the word's initial phoneme (voicing, frication, vowel length, consonant versus vowel, and the presence of a burst), following Balota et al. (2004).

Further, we added phonological controls not considered by Balota and colleagues: three measures for the $(\log )$ frequency of the initial diphone. We considered these measures with an eye towards capturing variance due to differential familiarity of the articulatory gesture for the first two phonemes. The first measure, the overall diphone frequency, was the cumulated frequency of the target word's initial two phonemes, calculated over all words and all positions in these words. This is our position-independent estimate. The other two measures were position-specific, one conditioned on being word initial, the other on being syllable-initial.

\section{Measures of frequency}

Our primary frequency measure was the surface frequency of the word as listed in CELEX. This frequency is based almost completely on written British English (Renouf, 1987). The surface frequency count was string based, cumulating over all forms (of any word category) that were identical to the form as listed in the Balota database. In our analyses, we entered log frequency into the model, for two reasons. First, lexical decision latencies have been reported to increase by a constant number of milliseconds for each $\log$ unit of frequency (Rubenstein \& Pollack, 1963; Scarborough, Cortese, \& Scarborough, 1977). Second, word frequencies are approximately log normally distributed (Baayen, 2001; Carroll, 1967), i.e., logarithmically transformed frequencies are approximately normally distributed. This is essential for parametric statistical techniques, given their sensitivity to outliers. (For all frequency 
variables, we added 1 before taking the log in order to avoid undefined values for words with a frequency of zero.)

A second frequency measure addressed potential differences in frequency in written as contrasted with spoken English. Rather than including a frequency measure for spoken language along with the written measure (which would introduce very high collinearity), we included the normalized difference between these measures as a predictor. We normalized the difference as the written frequency count is based on a corpus of 18 million words while the spoken frequency count is based on a smaller corpus of 5 million words, the demographic subcorpus of spoken English in the British National Corpus, henceforth BNC (Burnard, 1995):

$\log \left(f_{\text {CELEX }}+1\right)-\log (18)-\left(\log \left(f_{\mathrm{BNC}}+1\right)-\log (5)\right)$.

Note that, since $\log a-\log b=\log (a / b)$, this frequency difference is equivalent to the $\log$ of the ratio of the absolute (untransformed) frequencies. Therefore, we will henceforth refer to it as the written-to-spoken ratio.

In their regression analysis, Balota et al. (2004) compared a number of different frequency counts based on written English, and observed that the Zeno counts (Zeno et al., 1995) had the highest correlations with the dependent variables, substantially outperforming the CELEX counts, even though both counts were based on corpora of roughly the same size (17-18 million words). One likely reason that the Zeno counts were superior predictors is that the materials in this corpus were better tailored to the written language typically encountered by the American students participating in the experiment - the Zeno counts are based predominantly on textbooks and were explicitly collected as an aid for the educator, whereas the Cobuild corpus on which the CELEX counts were based was designed for more general lexicographic goals (Renouf, 1987). In other words, what may be at stake here is a difference in written registers.

There is another register difference, however, that might be at least equally important: the difference between spoken and written language (Biber, 1988, 1995). We therefore investigated the $R^{2}$ values for regression models including only our surface frequency count (using CELEX) and our written-to-spoken frequency ratio as predictors for the latencies of the young age group. For visual lexical decision, the $R^{2}$ was 0.482 , outperforming the $R^{2}$ reported by Balota for the Zeno counts by some $12 \%$. For word naming, the $R^{2}$ was 0.108 , marginally outperforming the correlation for the Zeno counts by some $2 \%$. For both visual lexical decision and word naming, our measures outperformed all other frequency predictors graphed in Fig. 7 of the Balota et al. (2004) study (p. 292), including subjective frequency estimates. These analyses support our intuition about the importance of experience with the spoken language, and suggest that the superiority of the Zeno counts compared to the CELEX counts might indeed reside in the Zeno counts being based on a form of written English that approximates spoken English more closely in terms of its vocabulary. In what follows, we have therefore used the CELEX counts as a measure of written frequency in written registers of English, combined with the written-to-spoken frequency ratio as a means of controlling for register.

\section{Semantic measures}

As a measure of a word's number of meanings (cf. Jastrzembski, 1981), we considered the number of different synsets in which it is listed in WordNet (Beckwith, Fellbaum, Gross, \& Miller, 1991; Fellbaum, 1998; Miller, 1990). A synset in WordNet is a set of semantically related words. A synset instantiates the same core lexical concept, somewhat like a thesaurus, but more tightly constrained semantically. Examples of synsets are

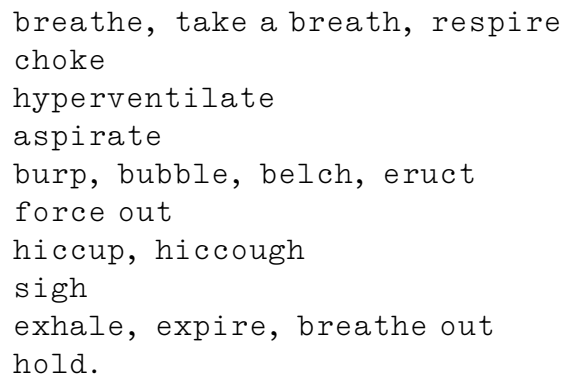

A word may appear in several synsets, once for each of its different meanings. The noun book, for instance, appears in synsets such as \{daybook, book, ledger\}, \{book, volume\}, \{script, book, playscript\}, and \{record, recordbook, book\}. A word may also appear as part of a spaced compound (a compound written with intervening spaces) in WordNet, as in the synset \{ledger, leger, account book, book of account, book\} (here, leger is an obsolete form of ledger). We kept separate counts of the numbers of synsets with the word itself (the simple synset count) and the numbers of synsets in which the word is part of a spaced compound (the complex synset count). Because Balota et al. (2004) studied a general connectivity measure based on WordNet, one interest of ours was to see to what extent morphological and non-morphological synonym-based connectivity might have distinguishable effects on lexical processing. (Note that the number of non-spaced compounds, e.g., bookcase, is incorporated in the family size measure.) 


\section{Morphological measures}

A first morphological distinction that we introduced into the analysis is whether a word is a noun or a verb as given in the CELEX lexical database. To remove potential arbitrariness in the CELEX assignments, and in order to take into account that the likelihood of verbal or nominal use (or the likelihood of morphological conversion) varies from word to word, we added the ratio of the frequencies of the nominal and verbal readings, henceforth the noun-to-verb ratio, as a covariate. (This measure is one of the predictors for whether a verb is regular or irregular, see Baayen \& Moscoso del Prado Martín, 2005 \& Tabak et al., 2005).

Three additional morphological variables were included: the word's morphological family size, its derivational entropy, and its inflectional entropy. These measures were already discussed in detail in the introduction. Here, we point out, first, that the logarithm of the family size count and the derivational entropy are highly correlated, and in fact identical when all family members are equiprobable, and that we calculated morphological family sizes and entropies on the basis of the morphological parses available in the CELEX lexical database.

To our knowledge, inflectional entropy is the only measure in the literature to address the complexity of a word's inflectional paradigm. Inflectional entropy was observed to be a significant independent predictor in visual lexical decision in (Tabak et al., 2005) (see also Traficante \& Burani, 2003, for the importance of inflectional families). Recall that our frequency measure is string-based, and does not collapse the frequencies of a word's inflectional variants into a 'lemma'-based frequency measure. Inflectional entropy offers a means to gauge the relevance of the inflectional variants, without having to increase collinearity by using a measure such as 'lemma frequency' side by side with 'surface frequency.'

\section{Method}

A problem that is encountered by anyone contemplating a multiple regression analysis of many lexical variables is that these variables tend to be interrelated. For example, word length and word frequency tend to vary together such that on average, longer words tend to appear less often in text. Similarly, words with stems that recur in many words and therefore have large morphological families tend to be relatively high in frequency. When many variables are correlated among themselves, i.e., when the predictors are highly collinear, severe problems for the interpretation of the regression models arise (see, e.g., Chatterjee, Hadi, \& Price, 2000).
When the predictors in multiple regression are uncorrelated, each predictor accounts for a unique portion of the variance. In other words, when there is no collinearity, the explanatory value of each individual predictor can be properly assessed. This is not possible when the predictors are collinear. Together, collinear variables may explain nearly all the variance, but it is never clear what part of the variance is explained by which variable.

The problem caused by collinearity manifests itself in inflated estimates of the coefficients and their variances in the linear model (Hocking, 1996). With inflated coefficients, prediction becomes unreliable and sometimes even meaningless. High degrees of collinearity may even lead to problems with machine precision. Collinearity gives rise to an ill-defined estimation problem for which there is no good single solution. Addition or deletion of a single data point may lead to a substantially different model.

Collinearity is often diagnosed by inspecting the pairwise correlations of the predictors, with the rule of thumb that no pairwise correlation should be higher than 0.7 . However, collinearity may be present even when pairwise correlations are not significant (Hocking, 1996). The condition number provides a better diagnostic for collinearity (Belsley, Kuh, \& Welsch, 1980; Hocking, 1996). The condition number can be viewed as a measure of the extent to which imprecision in the data can work its way through to imprecision in the estimates of the linear model. Low collinearity is associated with a condition number of 6 or less, medium collinearity is indexed by values around 12 , and high collinearity is indicated by values of 30 and above. The lexical data set we analyze in the present study has a condition number of 134.8. (We have followed Belsley et al. (1980) when calculating the condition number for the uncentered but scaled data matrix including the intercept.) To understand the implications of this high condition number, let's allow ourselves the optimistic assumption that our variables are known to three significant digits. What this high a condition number indicates is that a change in the data on the fourth place (i.e., a non-measurable change in the data, e.g., a change at the $0.1 \mathrm{~ms}$ level when response latencies are measured with only millisecond precision) may affect the least squares solution of the regression model at the second place. Only the first digit is therefore trustworthy. Hence, estimated coefficients with values such as $0.01,0.04$, and 0.001 are all indistinguishable and effectively zero. This calls into question the validity of any straightforward application of multiple regression techniques (including partial correlation) to our data set, and raises the question as to how to proceed.

One possible solution is to select one of several highly correlated variables, possibly the one that shows the greatest correlation with the dependent variable under study. This is how Balota and colleagues dealt with a 
series of different frequency counts of written English. This procedure has a drawback, however. Consider an educational test for assessing aptitude for science courses. Scores for mathematics and scores for physics are generally highly correlated, but using only the scores for physics would lead to inappropriate prediction about those students who are good in math but bad in physics. We have therefore not made use of this possibility, except when we had theoretical reasons for preferring a given measure. For instance, as mentioned above, the effect of morphological family size on lexical processing has been gauged by means of a type count, and more recently by means of a token-weighted type count, the derivational entropy. The two measures address the same phenomenon, they are mathematically related (see Moscoso del Prado Martín \& Kostić et al., 2004, 2004), but as we believe the entropy measure to be better motivated theoretically, we have not considered the simple family size type count in our regression analyses. For similar reasons, we opted for using only the syllable-initial measure for the initial diphone, and discarded its highly correlated word-based variant.

A second possibility is to orthogonalize pairs or groups of variables. We adopt this solution for three clusters of variables in our study. Recall that in addition to morphological connectivity and the other semantic variables, we were interested in the explanatory potential of spoken versus written frequency counts. Counts for spoken and written English tend to be highly correlated, and including spoken counts along with written counts would lead to a substantial increase in collinearity. We therefore used the difference in log frequency between written and spoken English as our frequency variable for register differences. This is mathematically equivalent to considering the ratio of written to spoken frequency. Importantly, the written-to-spoken ratio is not highly correlated with written frequency $(r=0.072)$, and thus can be entered into the regression equation without a counterproductive increase in collinearity. (This variable has a roughly symmetrical, bell-shaped probability density, range -6.6 to 5.6 , mean 0.7 , median 0.7 .) We followed a similar procedure with respect to the nominal and verbal frequency counts. Instead of adding two new frequency variables, we considered the ratio of $\log$ nominal to log verbal frequency. Again, we obtained a variable, the noun-to-verb ratio, that is not highly correlated with written frequency $(r=.056)$. (This variable is slightly skewed to the left, range -12.4 to 10.1 , mean 1.4, median 1.6.)

For larger numbers of variables, more complex methods for orthogonalization are available. In our study, in which the focus of interest is on morphological variables, we wanted to control for effects of orthographic and phonological consistency. We had 10 different measures: the token and type counts of forward (spelling-tosound) and backward (sound-to-spelling) inconsistent words (enemies), the token and type counts of phonological and orthographic neighbors, and the token and type counts of the friends. Each of these measures captures different aspects of consistency, and many are intercorrelated. The condition number for just this set of 10 variables alone was high, 49.6, indicating a high risk of harmful collinearity. In this case, we used a dimension reduction technique, principal components analysis (PCA), to obtain a smaller number of orthogonal, uncorrelated predictors. With just four new variables, the first four principal components, we captured $93 \%$ of the variation among the original 10 predictors. Inspection of the loadings of the original variables on these components revealed that the first PC $(43.5 \%$ of the variance) contrasted forward enemies (number of words with different pronunciation for the same sequence of letters) with phonological neighbors (number of words that differ by a single phoneme); the second PC (22.2\% of the variance) contrasted friends (number of words with the same letter sequence and the same pronunciation) with backward enemies (number of words with the same pronunciation but a different spelling); the third PC $(19.0 \%$ of the variance) forward enemies and friends, and the fourth PC ( $8.2 \%$ of the variance) the token and type counts. It is these four (uncorrelated) PCs that we actually used in our regression analyses. Hence, our analysis could address various aspects of consistency without unnecessarily increasing the collinearity in the data matrix. The disadvantage of this technique is that in general the interpretation of the principal components is not always straightforward. For control variables, such as consistency in the present study, this does not pose a major problem.

When PCA does not sufficiently reduce collinearity, two statistical techniques that have been developed to deal with collinearity are principal components regression and ridge regression. Of these two techniques, the consensus is that principal components regression is superior (see, e.g., Hocking, 1996). In principal components regression (henceforth PCA-regression), principal components orthogonalization is applied to the full matrix of predictors. The resulting principal components are the new, uncorrelated, orthogonal predictors for the PCA-regression. In PCA-regression, only the most important principal components are used as predictors, typically those that explain at least $5 \%$ of the variance in the data matrix of predictors (cf. Belsley et al., 1980). By means of a back-transformation, the outcome of the regression analysis can be interpreted in terms of the original variables. We use this technique below to check that the results of a stepwise multiple regression for word naming were not distorted by collinearity.

In passing, we note that a hierarchical regression analysis in which blocks of variables are entered into the model one at a time, does not constitute a principled solution to the problem of collinearity as it fails to deal 
with either the collinearity within blocks of variables or the collinearity between blocks. It is useful for establishing whether a block of variables has explanatory value over and above preceding blocks of variables, but the estimates of the individual coefficients in the model run the risk of being unreliable.

We also note that the collinearity in the data that we studied is, due to the selected variables, different from the collinearity in the larger data set (involving a different selection of predictors) reported in Balota et al. (2004). Balota and Yap (personal communication) observed a lower but still substantial condition number for their data, which they note is due largely to intercorrelations among the control onset variables. They also observed some minor instability for the coefficients, but only for the model fitting the naming latencies. The collinearity for the data studied in the present paper is due especially to the inclusion of morphological variables, which, as we will show below, cluster tightly with word frequency.

The assumption of linearity in multiple regression may introduce even greater problems with the accuracy of a multiple regression model than does collinearity (Harrell, 2001). There are several ways to deal with the non-linear relations in regression. In the absence of theoretical considerations that suggest a particular functional form (e.g., an exponential or sinusoidal curve), we will focus on exploratory methods. One such method is to add polynomial terms to the regression equation, a procedure followed by Balota et al. (2004) in their nonmultiple regression analysis of word frequency. However, polynomials do not fit 'threshold' effects well, which, as we shall demonstrate below, are present in our data. A more flexible technique is to make use of restricted cubic splines (see, e.g., Harrell, 2001, pp. 16-24; Wood, 2006, pp. 121-133). In construction, a spline is a flexible strip of metal or piece of rubber that is used for drawing the curved parts of objects. In statistics and in physics, a spline is a function for fitting non-linear curves. This function is itself composed of a series of simple cubic polynomial functions defined over a corresponding series of intervals. These polynomials are constrained to have smooth transitions where they meet, the knots of the spline. The number of intervals is determined by the number of knots. Each additional knot adds a coefficient to the regression model. To capture more substantial non-linearities, one will need more knots. In other words, the number of knots determines the degree of smoothing. Restricted cubic splines are cubic splines that are adjusted to avoid overfitting for the more extreme values of the predictor. In our analyses, we used the minimum number of knots necessary to model nonlinearities, whenever non-linearities were found to be statistically significant. Formally, this is accomplished by testing whether each of the coefficients associated with each additional knot is statistically significant.

\section{Results}

\section{Lexical space}

Fig. 1 provides a graphical overview of the correlational structure of the full set of our predictors. We first obtained the correlation matrix for the predictors using Spearman's $\rho$. We squared the elements of this matrix, to obtain a similarity metric that is sensitive to many types of dependence, including non-monotonic relationships (cf. Harrell, 2001). The resulting similarity matrix was input to divisive hierarchical clustering. Divisive clustering has the advantage of bringing out the main clusters in the data more clearly (cf. Venables \& Ripley, 2003, p. 317). All statistical analyses in this study were carried out using R ( R Development Core Team, 2005), the present cluster analysis was carried out using the module 'cluster' (Rousseeuw, Struyf, \& Hubert, 2005).

Note that the measures for phonological and orthographic consistency as well as our orthographic and other phonological controls cluster in the lower part of the dendrogram. Interestingly, our semantic measures (derivational entropy, family size, the synset counts, inflectional entropy, and the noun-to-verb ratio) are all united in a single cluster at the top of the graph, together with frequency. (Since hierarchical clustering is notoriously dependent on clustering method and the distance measure used, we note that a similar clustering emerged from a principal components analysis when the predictors are plotted in the space spanned by the first two principal components. The clustering provided further support for our hypothesis that word frequency is more tightly related to semantic lexical variables than to formrelated lexical variables.)

What the cluster analysis captures, and what inspection of Table 4 of Balota et al. also reveals, is that objective frequency clusters primarily with measures of word meaning rather than with measures of word form. This finding is in line with the hypothesis of Bates et al. (2003), who, using picture naming, suggested that frequency is primarily a measure of conceptual familiarity. Based on these observations, one of the questions we address below is to what extent frequency might also emerge as capturing semantic rather than formal aspects of lexical processing in word naming and visual lexical decision.

\section{Visual lexical decision}

We conducted a stepwise multiple regression analysis on the visual lexical decision data with log reaction time as the dependent variable. We applied the logarithmic transformation in order to eliminate most of the skewness of the distribution of reaction times, and to reduce the risk of the model being distorted by atypical outliers. 


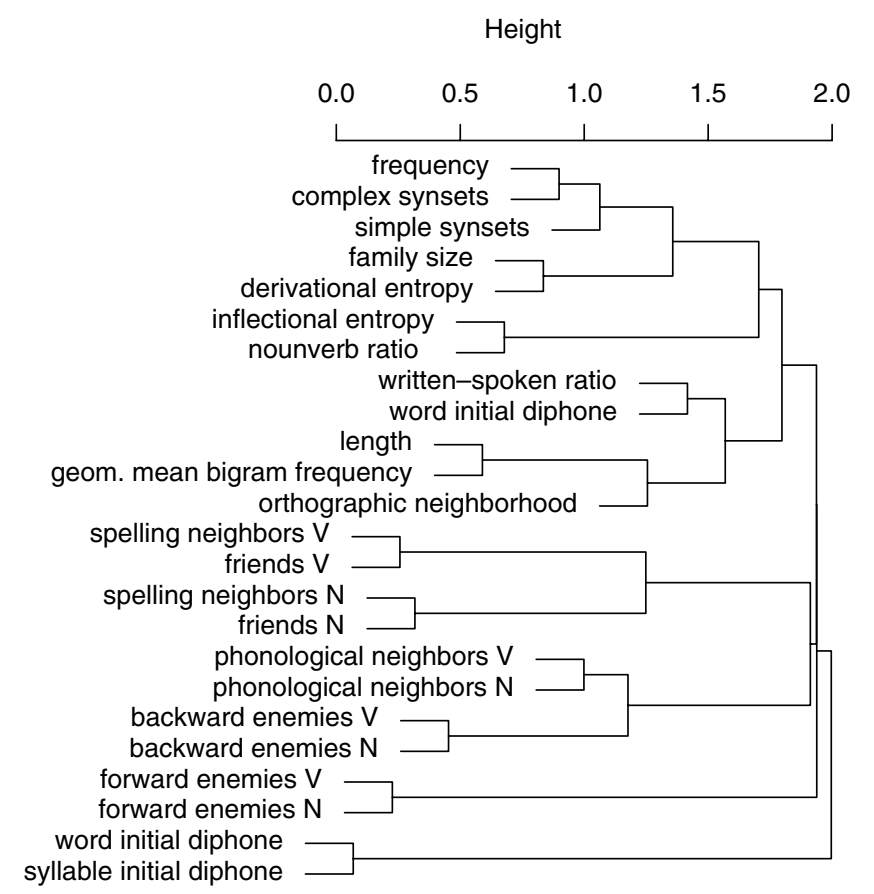

Fig. 1. Hierarchical cluster analysis (with divisive clustering) using Spearman's $\rho^{2}$ as a metric for the key predictors for visual lexical decision and word naming latencies.

Results are summarized in Fig. 2. Each panel represents the partial effect of one predictor. That is, we plot the effect of a particular predictor when all other predictors are held constant. Note that we report the non-linear as well as the linear components whenever the contribution of the non-linear components reaches a significance level of 0.05. Predictors that did not reach significance and were eliminated from the model are not depicted nor discussed any further. Inspection of the model for overly influential outliers through Cook's distance (a measure of overall leverage) and dfbetas (a measure of leverage with respect to the individual predictors), as well as through inspection of the standardized residuals, suggested the presence of 43 outliers $(1.9 \%$ of the data points). Outliers were defined as having a Cook's distance greater than 0.005 , an absolute standardized residual greater than 2.5 , or an absolute dfbeta greater than 0.2 . (Dfbetas quantify the change in the estimated coefficient if an observation is excluded, relative to its standard error.) Examples of outliers (as identified by the dfbetas) are jape, cox, cyst, skiff, broil, thwack, bloke, mum, nick, sub. The estimates of the coefficients differed minimally from those of the model for the complete set of data points $(r=.9999)$. In what follows, we restrict ourselves to the model obtained after removal of the outliers.

The upper left panel shows the effect of the first principal component based on our consistency measures $(F(1,2226)=8.58, p=.0034)$, the only principal compo- nent that emerged as significant. Overall, the consistency measures slowed decision latencies (all had positive loadings with this $\mathrm{PC}$ ). Slowing was greater for words with large phonological neighborhoods than for words with many feedforward enemies.

The second panel summarizes a small effect of whether the first phoneme of the word was voiced or voiceless, as this variable was observed to be significant by Balota et al. (2004). In our analysis, it reached significance as well $(F(1,2226)=8.56, p=.0035)$. As pointed out by Balota and colleagues, this suggests that the articulatory or phonological processes also codetermine lexical decision performance. The third panel depicts the effect of the geometric mean bigram frequency. This measure, which is strongly correlated with word length, was inhibitory $(F(1,2226)=11.29$, $p=.0008)$.

The upper right panel of Fig. 2 depicts the non-linear facilitatory effect of frequency $(F(4,2226)=299.85$, $p<.0001$; non-linear: $F(3,2226)=56.21, \quad p<.0001)$. The non-linearity asymptotes in a floor effect such that the facilitatory benefit of each additional log unit in frequency levels off as the limit of the fastest possible response is approached. Note that the use of restricted cubic splines is revealing here. If we would have used a quadratic term (as did Balota et al. (2004) in Fig. 8), the resulting regression curve would have suggested some slight inhibition for the highest frequencies, but this is just an artifact of using a technique that is known 

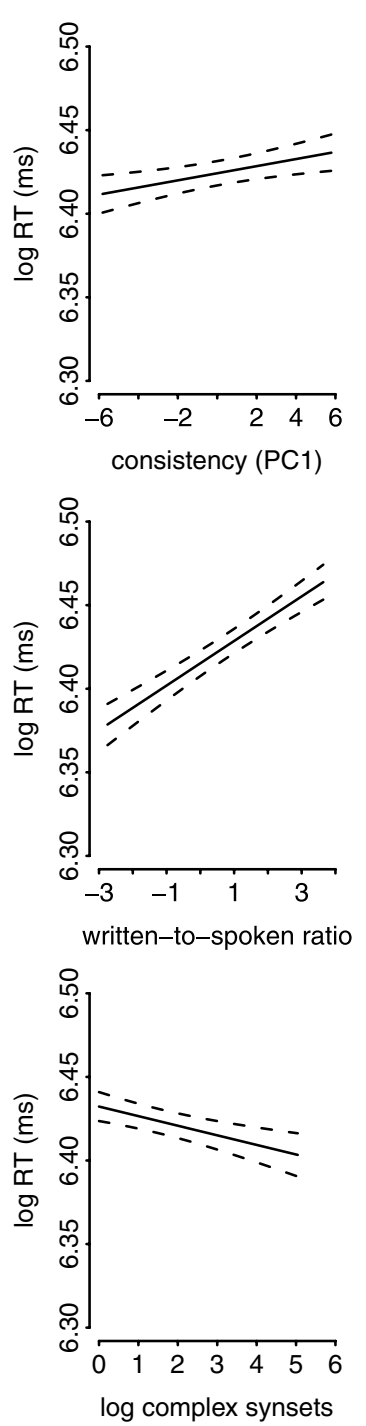
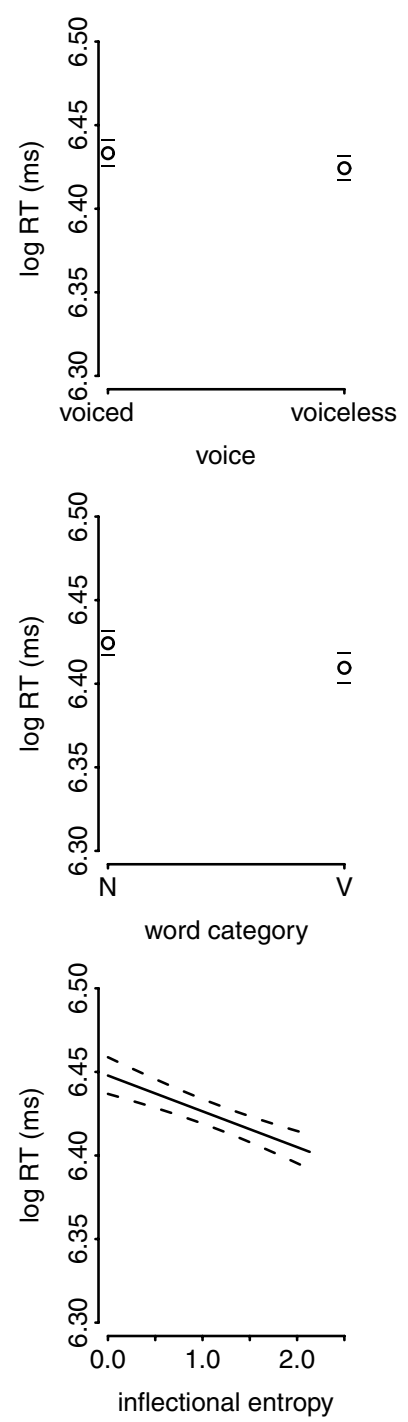
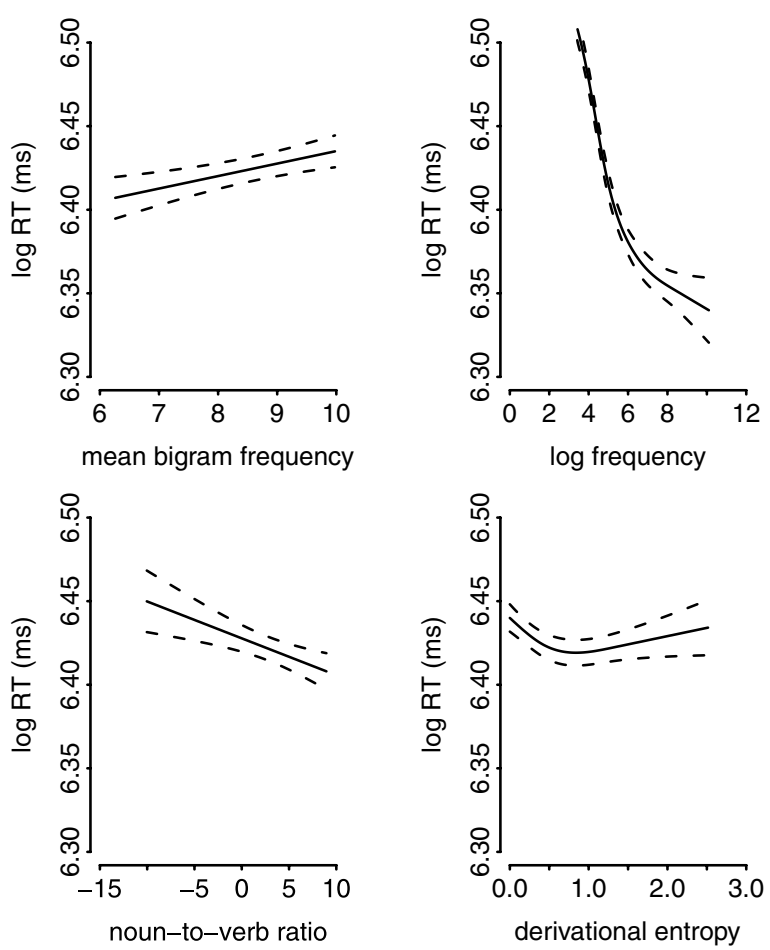

Fig. 2. Partial effects of the predictors for lexical decision latencies (adjusted for nouns and for the medians of the other covariates, $95 \%$ confidence intervals indicated by dashed lines).

not to work well for threshold effects such as the one we observe here.

The first panel on the second row represents the inhibitory effect of the written-to-spoken frequency ratio $(F(1,2226)=90.99, \quad p<.0001)$. As the discrepancy between a word's written and spoken frequency increases, response latencies in the visual lexical decision task increase. Words that occur predominantly in writing elicited longer response latencies in visual lexical decision than words that occur predominantly in speech.

The second and third panels address the role of word category. The central left panel shows that nouns elicited longer reaction times than did verbs $(F(1,2226)=11.62$, $p=.0007)$, and the central right panel shows that this effect was modulated by the noun-to-verb frequency ratio $(F(1,2226)=10.63, p=.0011)$. Stated simply, the more often a word is used as a noun rather than as a verb, the shorter its response latency.

The last three panels summarize the effects of the measures for lexical connectivity. Derivational entropy revealed a U-shaped curve, with initial facilitation followed by slight inhibition when morphological families were very large $(F(2,2226)=14.08, p<.0001$, non-linear: $F(1,2226)=19.01, p<.0001)$. The initial facilitation is consistent with the facilitatory effect of family size reported in earlier studies. The inhibitory effect for higher values indicates that apparently with very large morphological families performance might be disadvantaged, possibly because large families tend to encompass semantic heterogeneity (Moscoso del Prado Martín \& 
Bertram et al., 2004). The lower left panel reveals a facilitatory effect for the complex synset count $(F(1,2226)=$ $33.56, p<.0001)$. The last panel shows that increases in inflectional entropy were also facilitatory $(F(1,2226)=$ $13.56, p=.0002)$.

Fig. 2 also provides an overview of the effect sizes, as the vertical axis always shows exactly the same range of values for $\log R T$. Not surprisingly, frequency had by far the greatest effect, followed by the written-to-spoken ratio and inflectional entropy. Derivational entropy, the noun-to-verb ratio, and the number of complex synsets had small effect sizes, the effect of voice was tiny. Note that each of our measures of morphological connectivity had an effect size of the same order of magnitude as what for the purposes of the present study are control variables: the first principal component for consistency, geometric mean bigram frequency, and voicing. This highlights the importance of morphological connectivity in lexical decision along with better-studied variables such as consistency.

The condition number for the eight numerical predictors in this model was 41.15. The greater part of this condition number is due to including the geometric mean bigram frequency. Without this variable, the condition number reduces to 12.14 . We proceeded to inspect the stability of our model by conducting a resampling validation with 200 bootstrap runs in order to obtain an $R^{2}$ measure corrected for overfitting (see, e.g., Harrell, 2001). The $R^{2}$ on the training data over-estimated the $R^{2}$ on the test data by $0.6 \%$ (the so-called optimism, an estimate of the extent to which we overestimate $R^{2}$, and hence a measure of the degree of overfitting), and the resulting bias-corrected $R^{2}$ was $54.0 \%$. It is interesting to note that fast backward elimination of predictors left all 10 predictors in the model in 167 out of 200 bootstrap runs, removed one predictor in 25 runs, and two predictors in eight runs. In all, the resampling validation points to a robust and reliable model that compares favorably to a model including the 24 original variables (and voicing) in our database. For that model, the bootstrap-adjusted $R^{2}$ was only $45.6 \%$, with the modal number of predictors retained at 9 (for only 49 bootstrap runs). We point out that because there was astronomical collinearity among the total set of predictors (the condition number was 135), the more parsimonious model with lower collinearity had superior explanatory power.

A parallel logistic regression model on the accuracy data revealed significance for the same set of predictors, with similar functional relations (all $p<.0001, z$-tests on the coefficients), except for the voicing of the initial phoneme, $p=.0152$. In addition, a small non-linear effect of length was present $(p<.0001$, non-linear: $p=.0001)$, the increase in accuracy decreased slightly with increasing length. By contrast, our analysis of the response latencies did not support word length as a significant independent predictor of visual lexical decision latencies $(p>.1)$. This finding contrasts with the results reported in two other studies, Baayen (2005) and New et al. (in press), both of which describe a non-linear, U-shaped effect of length on visual lexical decision latencies. The former study (which made use of a less comprehensive set of predictors) was based not only on the responses of the young age group (as the present study) but also on those of the old age group. We therefore fitted the same statistical model to the response latencies of the older subjects, using the same set of words. In contrast to what we observed for the younger subjects, the partial effect of word length emerged as highly significant and non-linear $(p<.0001$, non-linear: $p<.0001)$. This shows that the non-linear effect of length is characteristic of older, and potentially more experienced, or possibly slower, readers. Apparently, the effect of word length is visible for the young readers only in the accuracy measure.

The study by New et al. considered a much larger data set including large numbers of polysyllabic and morphologically complex words. For a subset of 4000 monomorphemic nouns, they report a curvilinear effect of length as well. It is possible that with the increase in power due to the larger number of words considered, an effect of length emerges in their data. On the other hand, further research may yield additional insights. For instance, it is unclear from their study what the age of the subjects participating in the underlying experiments was. Second, New et al. considered a highly restricted number of predictor variables (printed frequency, number of syllables, and number of orthographic neighbors), and did not take variables capturing orthographic consistency and aspects of a word's morphological and semantic connectivity into account. Given the complexity of multidimensional lexical space, regression analyses will need to be based on comprehensive and overlapping sets of predictors. Otherwise, inconsistent results may ensue when the same large databases are studied by different groups of researchers.

These considerations lead to the conclusion that great care and methodological rigor is required when using large databases of response latencies, with respect to the subject groups included, with respect to the kinds of items selected for analysis, and with respect to the predictors they include.

Balota et al. (2004) reported two interactions involving frequency that are of special interest: an interaction of frequency by Length, and an interaction of frequency by Neighborhood Density. When we added these interactions to our model, we failed to detect any support for them. A fast backward elimination algorithm removed all terms involving neighborhood density and length in letters from the model. The absence of these interactions in our model and their presence in that of Balota et al. (2004) can be traced to their using linear multiple regression. These authors were aware of the 
non-linear relation between frequency and reaction time, as they modeled it by adding a quadratic term to a model regressing latency on frequency (see their Fig. 8). However, they did not incorporate this non-linearity in their multiple regression analyses, because (Balota and Yap, personal communication) they were concerned that including both interactions and non-linear terms would lead to instability in the regression equation (Cohen, Cohen, West, \& Aiken, 2003, pp. 299-300). No such concern is expressed in Harrell (2001), however. In fact, Harrell argues that prediction accuracy may crucially depend on bringing non-linearities into the model. Here we note that, as shown in (our) Fig. 2, there is a marked non-linearity in the partial effect of frequency even after the effect of all other predictors has been taken into account. We also note that the a-priori imposition of linearity for frequency leads to a loss in prediction accuracy: The goodness of fit of a model with a linear frequency effect and an interaction of Neighborhood Density by Frequency (now significant, $p=.0011$ ) is inferior to the model without this interaction but with a non-linear effect of Frequency (adjusted $R^{2}=0.51$ as compared to $R^{2}=0.54$ ). Since the model with the superior explanatory potential also validated well under the bootstrap, instability in the regression equation does not seem to be at issue for our data.

The statistical literature advises not to investigate interactions between numerical predictors, unless there are theoretical reasons for doing so, see, e.g., page 33 in Harrell (2001). On the other hand, interactions that are observed repeatedly may warrent further theoretical interpretation. For the visual lexical decision latencies, we observed two significant interactions that we mention here for completeness. The count of complex synsets entered into interactions with residualized familiarity (a measure introduced below, $F(1,2223)=23.32$, $p<.0001)$ and with inflectional entropy $(F(1,2223)=$ $14.92, p=.0001)$. In both cases, the facilitatory effect of number of complex synsets was somewhat attenuated for higher values of residualized familiarity and inflectional entropy. Including these interactions in the model did not lead to substantial changes in the other predictors.

The hierarchical regression analysis of Balota et al. also led to the conclusion that Neighborhood Density has a facilitatory effect on visual lexical decision latencies, in line with studies such as Andrews (1989) and Forster and Shen (1996). Our analysis, however, did not reveal a significant effect of Neighborhood Density. A principal components regression using the 24 original predictors likewise did not reveal an independent contribution for this measure $(p=.5864)$. Instead, it pointed to significant inhibitory effects of the spelling neighbors and spelling friends (both $p<.0001$ ) with which the neighborhood count enters into a strong correlation $(r=.475$ and $r=.437$, respectively). This suggests that the overall neighborhood density measure has nothing to contribute once more sophisticated measures of spelling neighborhood sizes are taken into account. It is noteworthy that the two consistency measures for neighborhood effects are both inhibitory in the principal components regression, which is in line with the inhibitory effect of the first principal component for our measures of orthographic consistency in our ordinary least squares model (correlation with neighborhood density: $r=.353$ ). In other words, more sophisticated measures of neighborhood density seem to indicate inhibition rather than facilitation. Addressing collinearity in a principled way may therefore help to reconcile the puzzle of the inhibitory effect of neighborhood density reported for French (Grainger, 1990, 1992; Grainger \& Jacobs, 1996) and the facilitatory effect of neighborhood density reported for English (Andrews, 1986, 1989, 1992, 1997). We leave the issue of potential differences across languages and spelling systems for future clarification, and turn to the analysis of the naming data.

\section{Word naming}

Fig. 3 presents an overview of the predictors for naming latencies that remained in our stepwise regression analysis. As in the analysis of the decision latencies, we inspected the model for outliers. Using the same criteria, 59 outliers with undue leverage were identified and removed. Removal did not affect whether predictors reached significance, but resulted in a tighter fit with more precise estimates for the coefficients.

The first two panels on the top row concern the factors controlling for the effects of the voice key: the presence of frication, bursts, and vowel length $(F(3,2206)=33.57, p<.0001)$ and voicing $(F(1,2206)=$ $76.29, p<.0001)$. Collectively, these voice key variables accounted for $19.6 \%$ of the variance in the naming latencies. As shown by Balota et al. (2004), a greater proportion of variance can be captured by including a larger set of variables. We were reluctant to include many such variables, as this would have increased the collinearity in our data matrix. For instance, whether the initial segment is voiced or voiceless is predictable in a logistic regression model from inflectional entropy ( $p=.0041)$, the first principal component of phonological consistency $(p<.0001)$, and derivational entropy $(p=.0537)$. Instead of adding additional phonological voice key controls, we opted for using initial diphone frequencies as further quantitative controls.

The next two panels show the partial effects of the controls for the initial diphone. The third panel on the top row shows the effect of the log frequency of the initial diphone, calculated over syllable-initial positions. The first panel on the second row shows the effect of diphone frequency calculated over all 

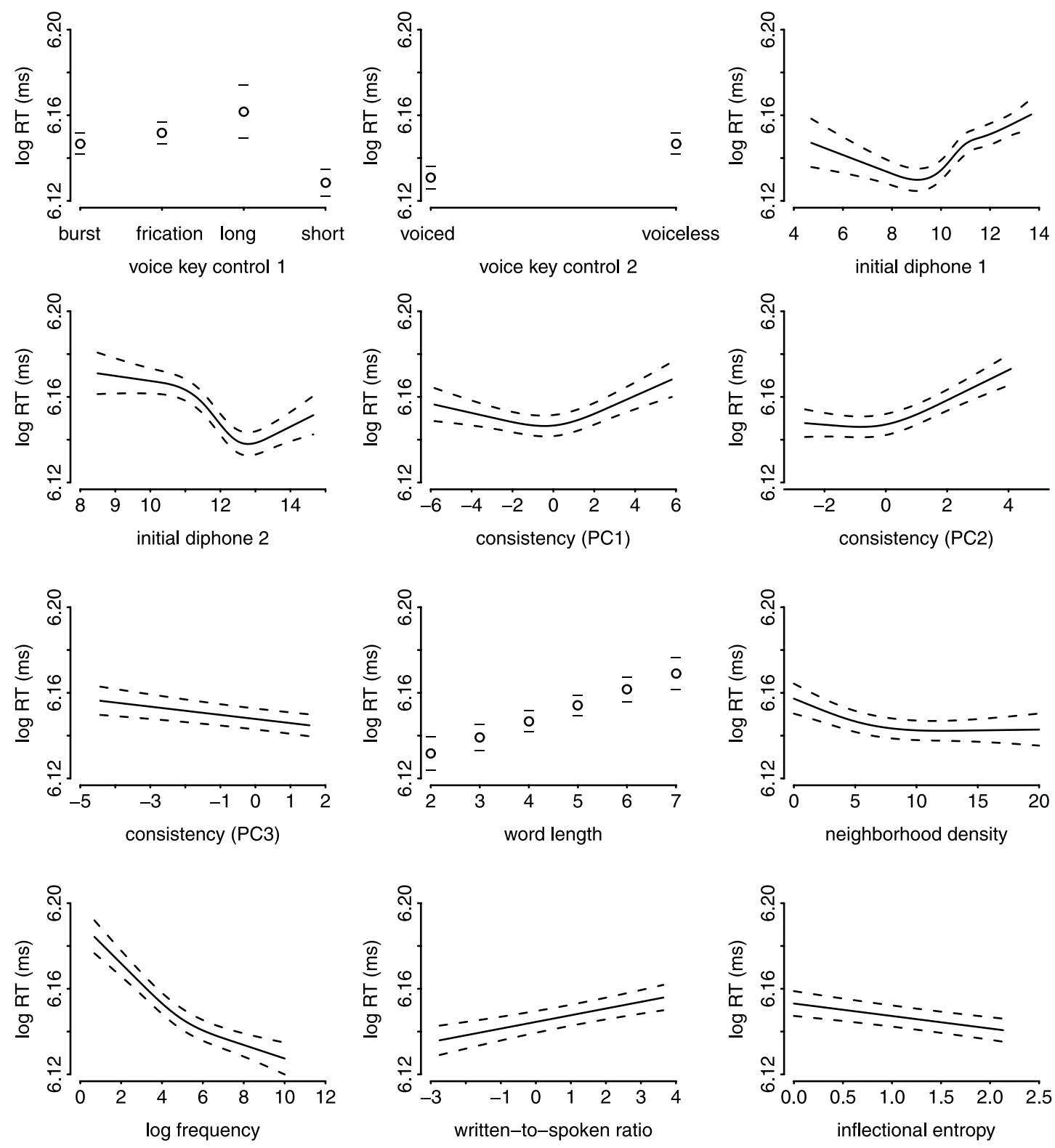

Fig. 3. Partial effects for the predictors for word naming (adjusted for nouns and for the medians of the other covariates, $95 \%$ confidence intervals indicated by dotted lines).

positions. Interestingly, the two measures are only weakly correlated $(r=.12)$, and both contribute independently to the model. Moreover, both show a marked U-shaped function, with initial facilitation followed by inhibition $(F(4,2206)=43.36, p<.0001$, non-linear: $F(3,2206)=23.09, p<.0001$, for the syllable-conditioned measure; $F(3,2206)=63.67, p<.0001$, non-linear: $\quad F(2,2206)=29.49, \quad p<.0001$ for the unconditional count). Possibly, the inhibitory effect of frequent initial diphones reflects a trade-off between articulatory ease (facilitatory) and costs of selection between other forms that begin with the same diphone.

The next three panels of Fig. 3 show the partial effects for three (out of four) principal components that represent the variables for orthographic consistency. The first principal component emerged with a U-shaped function, unlike in visual lexical decision, where it was linear $(F(1,2206)=22.17, \quad p<.0001$; non-linear: $F(1,2206)=31.57, p<.0001)$. Apparently, not only larger but also smaller numbers of phonological and spelling neighbors slow naming latencies. In addition, 
PC2 was mainly inhibitory $(F(1,2206)=28.95, p<.0001$; non-linear: $F(1,2206)=12.10, p=.004)$ and PC3 facilitatory $(F(1,2206)=17.37, p=.0001)$. More friends facilitated while more backward enemies inhibited (PC2), and the number of feedforward enemies inhibited while the number of friends facilitated (PC3). The fourth principal component, which pulled apart the type-based and token-based measures, was not significant.

Increases in word length (second panel on the third row) tended to slow naming as expected $(F(1,2206)=$ 52.01, $p<.0001)$. Orthographic neighborhood density was facilitatory, but tended to asymptote quickly $(F(2,2206)=9.08, p=.0001$, non-linear: $F(1,2206)=$ $7.90, p=.0050$ ), as can be seen in the last panel on the third row of Fig. 3.

The next two panels present two frequency effects. The effect of written frequency includes a small but significant non-linearity showing that it leveled off slightly for higher frequencies $(F(2,2206)=143.91, p<.0001$, non-linear: $F(1,2206)=13.95, p=.0002)$. Balota et al. did not observe such a non-linearity for naming, which may be due, first, to their use of a quadratic term (enforcing a specific functional form on the curve) where we used splines, and second, to our analysis including covariates whereas Balota and colleagues considered frequency by itself. The presence of this non-linearity and the similar form of this non-linearity in visual lexical decision supports our interpretation of a floor effectthe closer one gets to the fastest possible naming latency, the less the additional benefit of an additional log frequency unit is.

The contribution of the written to spoken frequency ratio that we observed for visual lexical decision also emerged for naming latencies. As depicted in the center lower panel, words used predominantly in written as contrasted with spoken English tended to have prolonged latencies $(F(1,2206)=26.46, p<.0001)$.

The last panel summarizes the effect of inflectional entropy, which was also facilitatory as in visual lexical decision $(F(1,2206)=15.75, p=.0001)$. None of the other morphological variables were significant predictors for the naming latencies. (Interactions of frequency by length and of frequency by neighborhood density did not reach significance. When the non-linear components are removed for frequency and neighborhood density, a significant interaction of frequency by neighborhood density is observed, but the interaction of frequency by length remains insignificant. The adjusted $R^{2}$ of this model was 0.49 , that of the simpler main effects model with non-linearities included was 0.50.)

Even for the present small number of predictors for the naming latencies, collinearity was quite high with a condition number of 138.85 . We therefore checked the validity of this model in two ways. First, we ran a principal components regression. In this regression analysis, all our predictors were retained as significant $(p \leqslant .001$ except for PC3, $p=.0537$ ), and the directions of the effects were in correspondence with those in Fig. 3.

Second, we validated the model with 200 bootstrap resampling runs. In 196 runs, all predictors were retained by a fast backward elimination algorithm, in four instances, one predictor was discounted. The optimism for the $R^{2}$ was $1.0 \%$, and the bootstrap-adjusted $R^{2}$ was equal to $49.1 \%$. As in our analysis of the lexical decision data, the explanatory power of the parsimonious model with 12 predictors was superior, albeit slightly, to a bootstrap-validated model including the 24 raw variables from our database $\left(R^{2}=41.1 \%\right.$. The mode of the number of variables retained across the 200 runs for all variables was 14 and this occurred in only 60 of the runs). We therefore conclude that the model summarized in Fig. 3 is both parsimonious and adequate.

A comparison of Figs. 3 and 2 reveals, first of all, that most of the semantic predictors that are significant for lexical decision are irrelevant for word naming. The greater sensitivity of visual lexical decision to semantic variables is well known (Katz \& Feldman, 1983; Lupker, 1979; Seidenberg \& McClelland, 1989). Nevertheless, the irrelevance for naming of word category, the noun-toverb ratio, derivational entropy, and the synset counts is remarkable, and suggests to us that the importance of word meaning is actually substantially reduced in simple word naming compared to lexical decision.

Note that the effect size of word frequency in naming was roughly twice that of the other predictors, all of which emerged with similarly sized effects. In lexical decision, the relative effect size of frequency was even larger. The greater relative effect size in visual lexical decision as compared to word naming is consistent with our intuition that a large part of the frequency effect captures semantic familiarity.

\section{Ratings and norms}

In their analysis, Balota et al. (2004) included subjective frequency estimates as a predictor in their multiple regression models for visual lexical decision and word naming latencies. In the light of Gernsbacher (1984), this is not surprising: Subjective frequency estimates would be superior estimates of frequency of occurrence compared to counts based on corpora. Moreover, Balota and colleagues' aim was to partial out any potential effects of frequency in order to be conservative with respect to their main goal, establishing the importance of semantic variables over and above form variables.

We were hesitant to include subjective frequency ratings as a predictor in the preceding analyses, because we had observed that measures other than frequency appear to predict subjective frequency ratings, see, e.g., Schreuder and Baayen (1997) and Balota et al. (2001). However, rigid the methodology by means of which subjective frequency ratings are solicited, they remain measures 
based on introspection, and we have no guarantee whatsoever that these estimates are uncontaminated by other variables. Furthermore, as we were interested in the unique explanatory potential of the individual predictors, including ratings would be counterproductive and lead to increased collinearity, since the ratings themselves enter into functional relationships with many of the other predictors.

To obtain evidence that subjective frequencies are an independent experimentally elicited variable in their own right, whose status is analogous to that of visual lexical decision latencies or word naming latencies, we studied the subjective frequency estimates in the Balota database with a stepwise regression analysis, using the same predictors as in the preceding analyses. The predictors that emerged as significant are summarized in Fig. 4. As for the analyses of decision and naming latencies, we identified potentially harmful outliers (51) on the basis of the initial analysis, and refitted the model to the reduced data set. As for the naming data, removal of outliers did not lead to different conclusions, but allowed for more precise estimates of the coefficients.
The upper left panel shows a nearly linear increase of the rating judgments with frequency $(F(3,2222)=$ 1548.20, $p<.0001$; non-linear: $F(2,2222)=6.56, p=$ $.0014)$. It will come as no surprise that words with greater frequencies elicited higher subjective frequency estimates. Interestingly, as shown by the next figure, the effect of subjective frequency was modulated by the written to spoken frequency ratio, such that a greater representation in written than in spoken English led to lower ratings $(F(3,2222)=143.76, \quad p<.0001$, non-linear: $F(2,2222)=6.90, p=.0010)$. This is reminiscent of the effect of the written-to-spoken ratio in lexical decision and word naming, where a greater ratio led to longer latencies. Evidently, the role of spoken frequency in studies of visual word recognition has been underestimated.

Of even greater interest are the remaining panels of Fig. 4. The first two central panels show the effects of word category. Nouns elicited slightly lower ratings than did verbs (left panel, $F(1,2222)=11.19, p=.0008$ ), although higher noun-to-verb ratios led to higher ratings (center panel, $F(2,2222)=7.38, p=.0006$, non-linear:
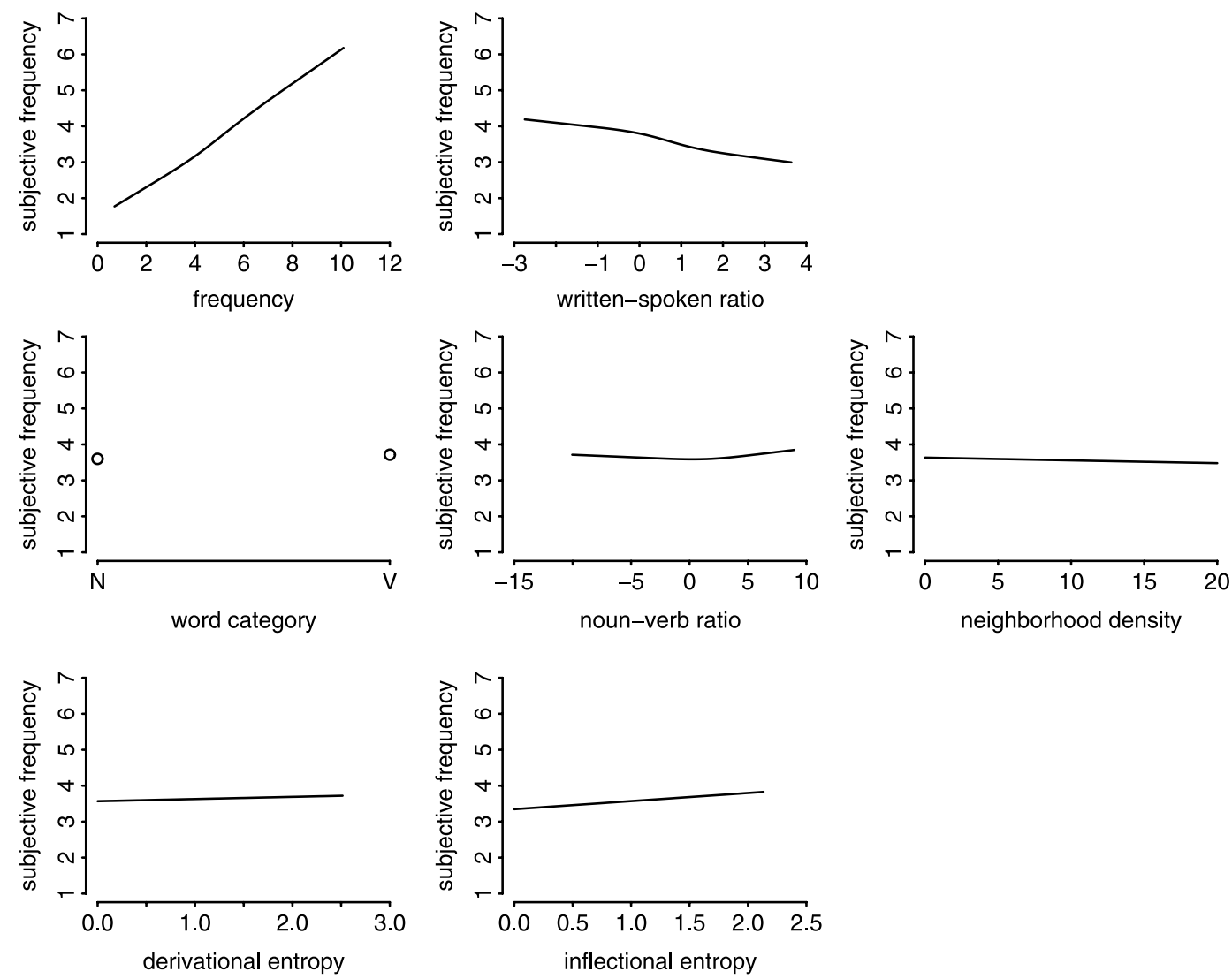

Fig. 4. Partial effects of the predictors in the multiple regression model fitted to the subjective frequency estimates. The graphs are calibrated for nouns and the medians of the numeric predictors. All 95\% confidence intervals were very narrow, and hence are not shown. 
$F(1,2222)=13.47, p=.0002)$. We leave it to the reader to verify that this is the expected mirror image of what we observed for visual lexical decision. The third panel captures the negative correlation between neighborhood density and the ratings $(F(1,2222)=12.28, p=.0005)$. This negative correlation is the flip side of the positive correlation observed for the first principal component for consistency.

The panels on the bottom row summarize the positive correlations of the two entropy measures with the ratings (derivational entropy: $F(1,2222)=4.89$, $p=.0270, \quad$ inflectional entropy: $F(1,2222)=68.18$, $p<.0001)$. Their predictivity is in line with the results reported in Schreuder and Baayen (1997) for the simple family size count. Note that the signs of the slopes of these effects are opposite to those in visual lexical decision, just as is the case for frequency: longer lexical decision latencies correspond with lower subjective frequency estimates. Thus, subjective frequency estimation emerges as a task that is in many ways the off-line inverse of visual lexical decision. By implication, if subjective frequency estimates are included as a predictor for other experimentally obtained dependent variables, it is crucial to first partial out the effects of the related linguistic predictors. We will return to this issue below. The bootstrap-validated $R^{2}$ of this model was $0.756-$ more than two thirds of the variance in the subjective ratings is predictable from 'objective' lexical measures.

\section{Age of acquisition and imageability}

Subjective frequency ratings are not the only experimentally elicited variables that have been reified as measures of lexical processing. In what follows, we will briefly touch upon two other such measures: age of acquisition norms, and norms for imageability, as made available by Bird, Franklin, and Howard (2001). These norms were available to us only for a small subset of the words in our database, and the results are therefore more provisional compared to those based on the subjective frequency estimates.

The upper two rows of Fig. 5 bring together the predictors for age of acquisition (shown on the vertical axis) that reached significance in a stepwise multiple regression analysis of a subset of 336 words for which we had at our disposal (a) age of acquisition norms, (b)
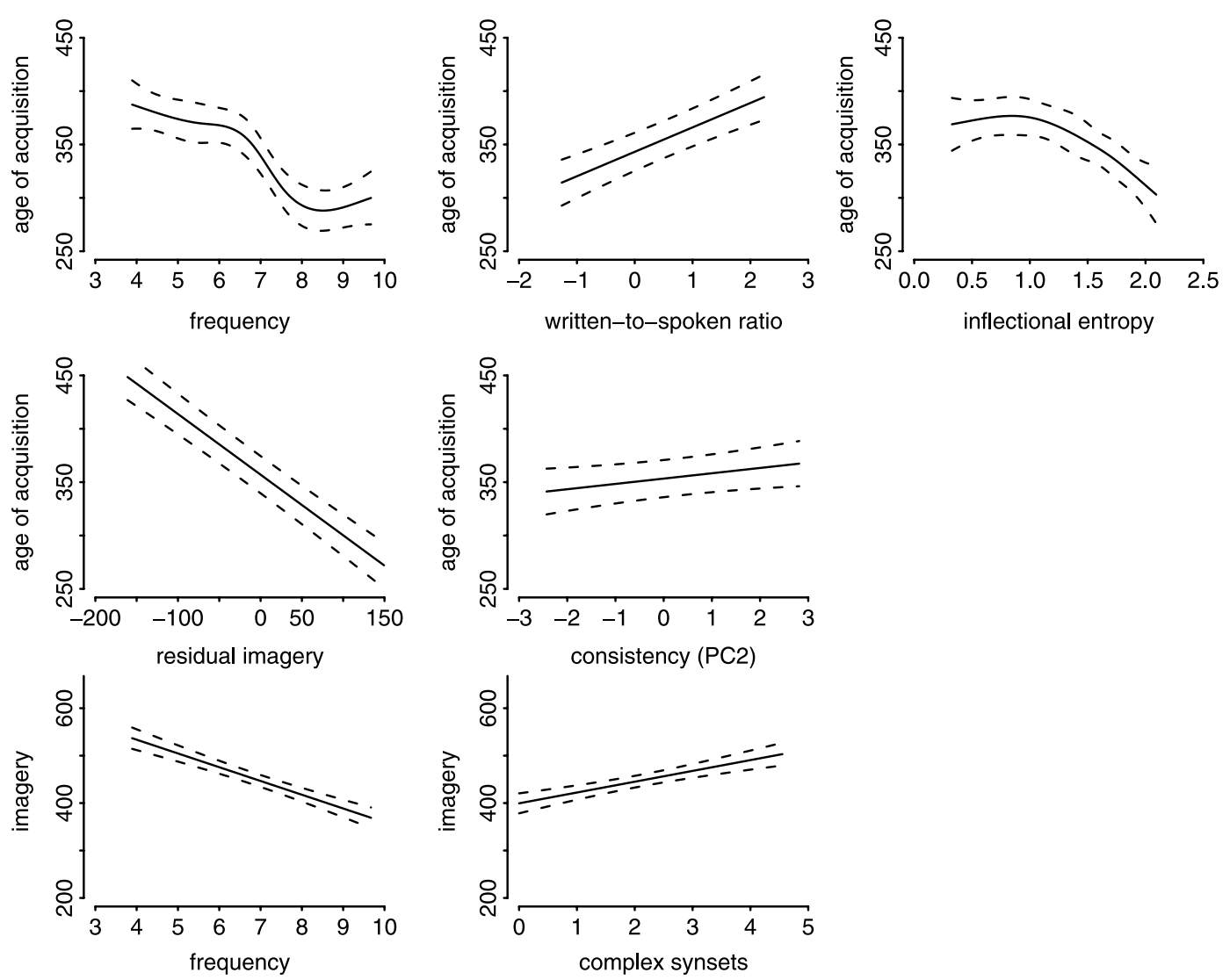

Fig. 5. Partial effects of the predictors in the multiple regression model for the subset of 336 words fit to age of acquisition norms (upper two rows) and to imageability norms (lower row). Dashed lines represent $95 \%$ confidence intervals. 
imageability norms, and (c) the association norms from the Florida database made available by Nelson et al. (1998). As in previous figures, the panels display the partial effects of the predictors, i.e., their effect when the other predictors in the model are held constant. Greater values on the vertical axis denote an older age of acquisition.

Not surprisingly, frequency and age of acquisition were negatively correlated $(F(4,324)=22.85, p<.0001$, non-linear $F(3,324)=5.18, p=.0017)$ : Less frequent words are acquired later in life. The written-to-spoken ratio showed a positive correlation $(F(1,324)=40.33$, $p<.0001)$ such that words that appear predominantly in writing tend to be learned later than words that are predominant in speech, which makes sense as well.

The upper right panel shows that words with a greater inflectional entropy are learned earlier $(F(4,324)$ $=8.87, \quad p<.0001$, non-linear: $F(3,324)=3.17, \quad p=$ .0245). Apparently, words with many inflectional variants, and inflectional variants that are all used frequently, are acquired earlier in life.

The first panel on the second row shows the linear relation of imageability (or more precisely, residual imageability, see below) and age of acquisition $(F(1,324)=189.18, p<.0001)$. Not surprisingly, more imageable nouns are learned earlier. Finally, orthographic consistency (as captured by the second principal component in our reduced space) revealed a positive correlation with age of acquisition $(F(1,324)=4.34$, $p=.0381)$. Words with more friends and fewer backward enemies are acquired earlier.

The bootstrap-validated $R^{2}$ for this model was $51.2 \%$, indicating that half of the variance in the age of acquisition norms that were available to us can be predicted from other lexical variables and imagery.

The two panels in the third row of Fig. 5 address respectively the predictivity of frequency $(F(1,333)=$ 83.93, $p<.0001)$ and the count of complex synsets $(F(1,333)=32.46, p<.0001)$ for the imageability norms (shown on the vertical axis) as revealed by a stepwise multiple regression analysis. Higher-frequency words tended to have lower imageability (e.g., do, quite, until), whereas words occurring in many complex synsets (e.g., plant, fish, box) tended to be more imageable. The bootstrap-validated $R^{2}$ for this model was $19.2 \%$.

The correlational involvement of subjective frequency ratings and measures of imageability and age of acquisition with other lexical variables should induce caution against adding these variables as predictors into models for chronometric (or other) measures of lexical processing. Inclusion would increase collinearity and lead to potentially unstable models and obscure interpretation. The solution that we adopt is to focus on the residuals of the regression models. Each residual represents the part of subjective frequency, age of acquisition or imageability that cannot be predicted from the lexical variables in our database.
Fig. 6 shows how the residuals of the rating-based measures, as well as three semantic association norms from the database of Nelson et al. (1998) cluster with the other variables in our new regression analysis. We retain Spearman's $\rho^{2}$ as distance measure and apply it to our data set of 336 words. Interestingly, the upper cluster brings together all measures pertaining to 'semantic' properties that do not relate to form (e.g., family size, synset measures, imageability, and connectivity) as well as subjective and objective frequency, the only exception being the word initial diphone frequency. Further down in the dendrogram we find all measures for a word's form. This cluster analysis is consistent with the grouping depicted in Fig. 1. The pattern supports our claim that frequency is more closely affiliated with variables pertaining to morpho-semantic connectivity than to measures of word form.

In a final series of analyses, we addressed the explanatory potential of the three residualized measures for the chronometric measures. First we considered the full data set, to which we added the residuals for the subjective frequency ratings as a new predictor.

When we added the residual subjective frequency rating to the model for lexical decision, there was a large and significant effect $(F(1,2220)=186.43, p<.0001)$, such that the higher (residual) ratings led to shorter visual lexical decision latencies, as expected. The bootstrapvalidated $R^{2}$ for this model was $57.6 \%$ (as compared with the original bootstrap-validated $R^{2}$ of $53.8 \%$ reported above). The implication is that the subjective frequency rating is a powerful variable in its own right, even after the lexical covariates that co-determine this subjective measure have been properly partialled out. The residual rating also contributed to explaining the naming latencies $(F(1,2211)=15.60, p=.0001)$, but led to only a tiny increase in the bootstrap-validated $R^{2}$, an increase from $48.3 \%$ to $48.8 \%$. The other predictors in these two models were only minimally affected. Summing up, residual subjective frequency estimates are more predictive for visual lexical decision than for naming. Given that visual lexical decision is more sensitive to semantic variables than is word naming, this finding strongly suggests that subjective frequency estimates are more sensitive to semantic familiarity than to form familiarity.

Finally, we selected the predictors that were significant in the model fitted to the full data set and supplemented them with the three residualized predictors along with the three association measures from the Florida database that were available to us for the smaller data set. When we conducted stepwise regression analyses on this smaller data set composed of 336 words, the only new variable that exerted an effect was residual imageability on lexical decision latencies $(F(1,329)=10.80, p<.0011)$. Moreover, variables such as the written-spoken ratio, the noun-verb ratio, and 


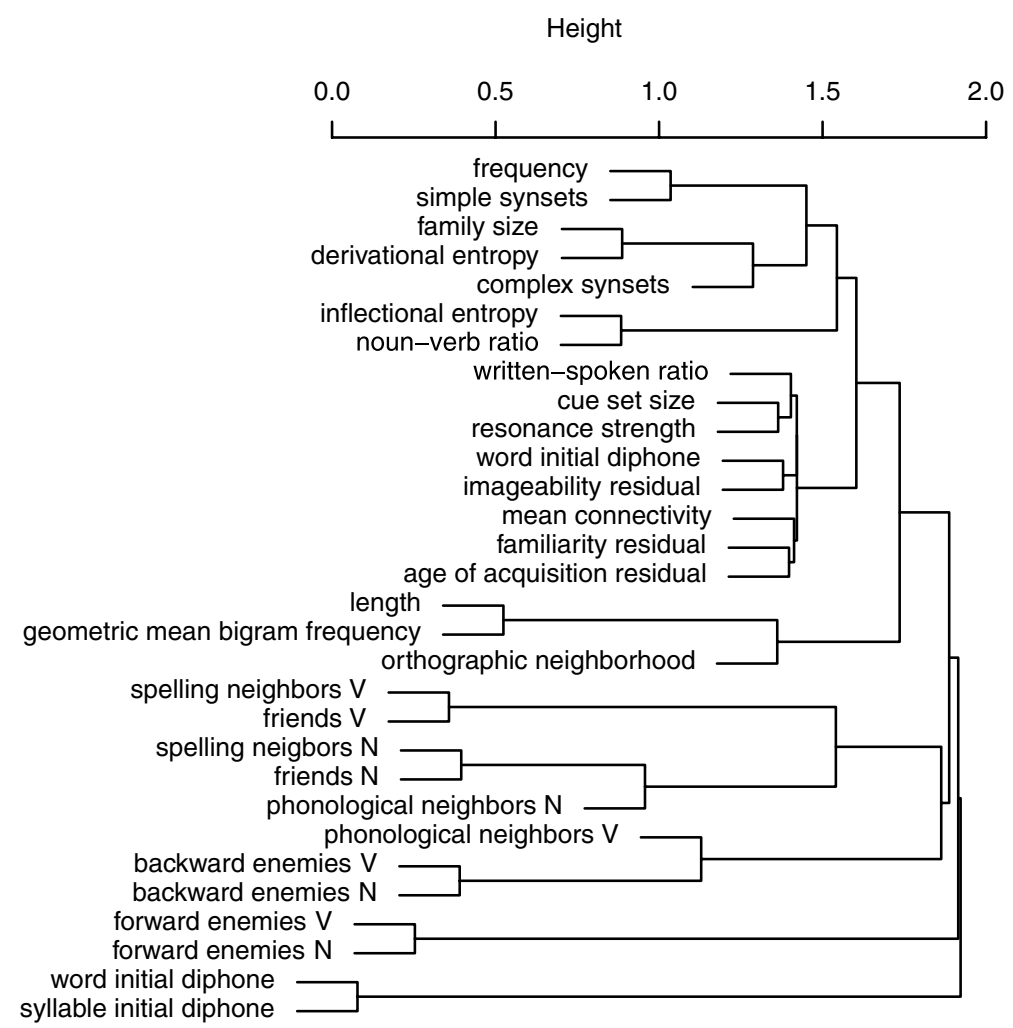

Fig. 6. Hierarchical cluster analysis (with divisive clustering) using Spearman's $\rho^{2}$ as a metric for the enlarged set of predictors for our subset of 336 words.

inflectional entropy remained significant for this smaller data set of decision latencies. Importantly, age of acquisition did not. From this, we infer that a properly residualized measure of age of acquisition is unlikely to lead to substantial changes in the models that we obtained for the full data sets.

\section{General discussion}

The main goal of the present study was to gauge the importance of morphological measures as well as frequency in the lexical processing of morphologically simple words by means of a regression analysis on the by-item latencies of the young participants in the visual lexical decision and word naming experiments made available by Balota and his colleagues (Balota, Cortese, \& Pilotti, 1999; Spieler \& Balota, 1998). This study therefore extends the seminal study of Balota et al. (2004) by considering a number of morphological variables that they did not consider in their analyses. Our primary finding is that we see the new morphological variables of inflectional and derivational entropy at work in visual lexical decision, each with effect sizes that are at least as large as that of the single form variable (orthographic consistency) that remained signifi- cant in a stepwise modeling procedure. In addition, we observed a special role for spoken frequency. In word naming, by contrast, the only morphological variable that retained the status of a significant predictor was inflectional entropy.

Table 1 lists for each significant predictor a lower bound for the percentage of variance accounted for by that predictor. These percentages were computed by comparing the adjusted $R^{2}$ of the full model with the adjusted $R^{2}$ of that model after removal of the predictor. For word naming, for instance, the $R^{2}$ of the full model was 0.4999. Removal of frequency reduced the $R^{2}$ to 0.4391 , hence the percentage of variance uniquely accounted for by frequency over and above all other predictors is 6.08 .

Table 1 is revealing in that it shows unambiguously that the unique contributions of many individual predictors is minute, even though their presence in the model is justified statistically. Considered jointly, the total amount of variance that we can attribute uniquely to specific predictors is roughly half of the total amount of variance captured by the models $\left(R^{2}=0.4999\right.$ for naming, $R^{2}=.5771$ for visual lexical decision). This is a consequence of the high collinearity characteristic of lexical space.

Note, further, that the unique contribution of frequency is substantially reduced in naming compared to 
lexical decision. Moreover, morphological variables such as Derivational and Inflectional Entropy account for more unique variance than established measures such as orthographic consistency or number of meanings (as captured by the count of complex synsets). The implication is that measures of morphological connectivity play an important role in lexical processing of monosyllabic morphologically simple words.

Fig. 7 complements Table 1 with a visual summary at the level of groups of variables. The top panel represents the additional amount of variance captured when five different groups of predictors - voice key controls, form variables, frequency variables (written frequency and written-to-spoken ratio), semantic variables (including morphological variables and noun-verb ratio), and residual familiarity - are entered sequentially into the regression equation. Visual lexical decision is in white, and word naming in black. In the latter, the minor roles of frequency, meaning, and familiarity contrast with the large contributions of the voice key controls and form variables. Conversely, the form variables in visual lexical decision are dwarfed by the enormous contribution of
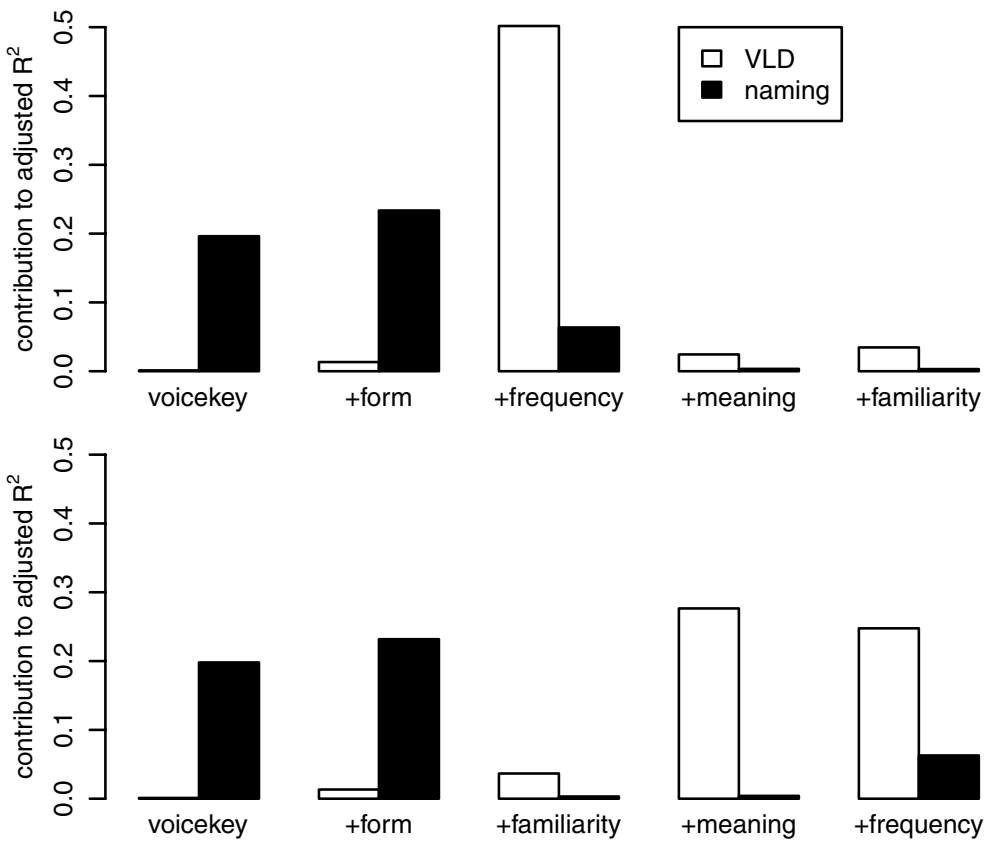

Fig. 7. Contribution to the adjusted $R^{2}$ for visual lexical decision (white bars) and naming (black bars) as the groups of variables shown on the horizontal axis are added successively to the model from left to right (upper panel). The lower panel shows the contributions when semantic variables are entered before frequency.

Table 1

Lower bounds for the percentage of variance (adjusted $R^{2} \times 100$ ) explained by the significant predictors in word naming (left) and visual lexical decision (right)

\begin{tabular}{lllr}
\hline Word naming & & & \multicolumn{1}{c}{ Lexical decision } \\
\hline Frequency & 6.08 & Frequency & 24.69 \\
Initial diphone 2 & 4.33 & Residual familiarity & 3.46 \\
Initial diphone (syllable-based) & 3.88 & Written-to-spoken ratio & 1.82 \\
Frication/Length first phoneme & 2.24 & Inflectional entropy & 0.70 \\
Voicedness first phoneme & 1.73 & Derivational entropy & 0.48 \\
PC2 Consistency & 1.28 & Complex synsets & 0.25 \\
Length & 1.14 & Word category & 0.22 \\
PC1 Consistency & 0.91 & Noun-to-verb ratio & 0.20 \\
Written-to-spoken ratio & 0.59 & Mean bigram frequency & 0.17 \\
Neighborhood density & 0.36 & Voicedness first phoneme & 0.13 \\
PC3 Consistency & 0.36 & PC1 Consistency & \\
Inflectional entropy & 0.34 & & \\
Residual familiarity & 0.31 & & \\
\hline
\end{tabular}


frequency. Moreover, the role of form in visual lexical decision is also smaller than that of the semantic variables and the residualized familiarity measure.

We can alter the order in which we enter blocks of variables. In the upper panel, frequency preceded the semantic variables and residual familiarity, and provides us with a lower bound for the contribution of the semantic variables, and an upper bound for frequency. Because our frequency measures and our semantic measures are all correlated, the order in which the variables are entered into the model becomes crucial. The lower panel of Fig. 7 shows the contributions to the adjusted $R^{2}$ when we add frequency last, and add residual familiarity before the semantic measures. As residual familiarity is uncorrelated with the other measures, its contribution is virtually unchanged by order. Of principal import is that the upper bound for the semantic measures in lexical decision is much higher, and of the same order of magnitude as the lower bound for our frequency measures. For naming, nothing much changes as the contribution of semantic variables is minimal in this task.

Collectively, in visual lexical decision, measures of morphological connectivity such as derivational and inflectional entropy, number of complex synsets, and the noun-to-verb ratio (capturing aspects of morphological conversion) are predictors that jointly are, at a minimum, at least as important as measures of word form. At best they are equally as explanatory as the word frequency effect itself. Finally, there was no independent predictivity from the simple synset count. The implication is that a word's morphological connectivity rather than a word's number of morphologically unrelated synonyms emerges as the primary semantic factor in visual lexical decision.

In this study, we have demonstrated some of the problems that the reification of subjective estimates, whether of frequency, age of acquisition, or imageability can introduce. For instance, subjective frequency in and of itself is a dependent variable that is to a large extent (bootstrap-validated $R^{2}=.76$ ) predictable from other lexical measures (see also Balota et al., 2001). For subjective estimates of age of acquisition, roughly half of the variance could be traced to lexical predictors and imageability, and for subjective measures of imageability, it was the case that one fifth of the variance could be explained. For the subset of the data for which we had age of acquisition norms, the written-to-spoken ratio turned out to be predictive in lexical decision to the exclusion of (residualized) age of acquisition. For larger data sets, however, it may be that age of acquisition emerges as an independent predictor as well (De Jong, 2002).

While subjective frequency has a high correlation with the decision latencies $(r=-.67)$, partialling out the lexical predictors reduces this correlation to $r=-.20$, which amounts to a drop from $44.9 \%$ to a mere $4.1 \%$ of variance explained. This small contribution also is evident in Fig. 7. Balota et al. (2004:311) argued that their young subjects appeared "to have good metacognitive insights into their frequency of exposure to words." Our findings suggest another interpretation. Rather than claim that introspection provides access to frequency of exposure, subjective frequency estimates may be determined largely by the same variables that predict visual lexical decision latencies. In essence, subjective frequency estimates are contaminated, or enriched, by measures of morphological connectivity, as well as by the written-to-spoken ratio. The finding that in the regression a higher written frequency leads to lower instead of higher subjective estimates means that it is unlikely that participants are able to access the frequency with which they have encountered a word in written form. Although they might be tapping into the frequency with which they have encountered the word in spoken form, our preferred interpretation is that they are guided by conceptual familiarity. In support of our claim, we observed that residualized familiarity clustered with semantic variables, not with measures of word form (see Fig. 6).

We reiterate that both objective and subjective frequency cluster with semantic measures, including measures for morphological connectivity. What are the implications of this observation for the more general question of what is captured by frequency of occurrence? Balota and Chumbley (1984) and Balota et al. (2004) have shown that visual lexical decision is sensitive to more than aspects of a word's form. The results from the present study are consistent with this view, but invite a more extreme position, according to which in visual lexical decision frequency reflects primarily conceptual familiarity. Even though frequency (or rank) might still be a guiding principle for lexical access, as argued by Murray and Forster (2004), we have several reasons for pursuing the claim that frequency is a far more important organizational principle at the semantic level.

Not only is it unlikely that word frequency would primarily tap into formal levels of representation and processing given the structure of lexical space, it is the case that the gain in predictive accuracy by adjusting for the frequency of use in speech rather than in writing (by means of the written-to-spoken ratio) also argues against locating the frequency effect primarily at the level of visual access. The persistance of frequency effects in reading across changes in font (Allen, Wallace, \& Weber, 1995) provides further evidence for this position.

Furthermore, we have demonstrated that frequency is an important predictor for imagery, for age-of-acquisition, and for subjective frequency estimates. In essence, frequency is highly correlated with a number of variables that have no immediate relation with the familiarity of a word's orthographic form. Whenever frequency is varied, these variables will tend to covary. 
The reduction in the explanatory power of the frequency effect illustrated in Fig. 7 that results from partialling out the effect of established semantic variables provides further support for the tight relation between frequency and semantics. In fact, the naming data suggest an upper bound for the extent to which frequency reflects form-based processing. Given that word naming combines visual perception with speech production, there are two levels at which form representations can be involved, at the input and at the output levels. The combined form frequency effects at these two levels apparently contribute less than $10 \%$ to the explained variance. This is an upper limit, as we cannot exclude the possibility that meaning (and concomitant frequency-driven processing) was involved during naming.

Finally, the frequency effect was modulated by the noun-to-verb ratio. Conversion pairs such as thelto work share the same form, and nevertheless the frequencies with which they are used as a noun or as a verb are reflected in visual lexical decision latencies, see also Feldman and Basnight-Brown (2005). This cannot be explained in terms of properties of the word's orthographic form.

In addition to insights into the semantic nature of the frequency effect, the present study also offers a methodological advance. It illustrates the advantages of regression with restricted cubic splines to model nonlinearities. Serious consideration of non-linearities is an absolute prerequisite for accurate prediction (Harrell, 2001), even when there are no immediate, self-evident explanations available. For instance, we observed Ushaped non-linear relations in both visual lexical decision and in naming, for which we could offer only tentative explanations in terms of conflicting constraints. Obviously, further research is required here because we have also illustrated how spurious interactions may arise if non-linearities are not properly brought into the model.

The present study also called attention to the dangers of collinearity and suggested some simple strategies to address these dangers. The models that we presented were both economical and superior in predictivity to models including many more collinear variables.

In summary, our analysis fully supports the conclusion reached by Balota and colleagues that in visual lexical decision semantic factors are predictive over and above frequency and measures of a word's form. We have extended their work by showing that a series of morphological variables - variables for which we have independent evidence that they are semantic in nature - are also co-determining visual lexical decision latencies over and above measures of a word's form properties. We have taken their approach a step further by arguing that in visual lexical decision, word frequency itself is a measure that captures primarily semantics rather than word form, for instance, by showing that higher spoken frequencies correlate with shorter reaction times in visual lexical decision. Our analysis diverges from that of Balota and colleagues with respect to the interactions of word frequency by length and neighborhood density, which turn out not to be necessary once the non-linear nature of the frequency effect is brought into the model for visual lexical decision.

To conclude, we offer a final insight into the implications of the high collinearity of measures of lexical processing for theories of the mental lexicon. In our statistical analysis, we invested in reducing collinearity in order to ascertain whether our key variables are actually predictive independent of the other variables. This does not imply, however, that we think these key variables are truly separable in lexical processing. Rather, they capture aspects of a system that is characterized by high degrees of interconnectivity. This interconnectivity can be modeled by distributed mappings between orthographic, phonological and semantic representations, as in, for example, the triangle model (Harm \& Seidenberg, 2004; Seidenberg \& Gonnerman, 2000). In these models, storage is superpositional, and frequency affects the interconnectivity of nodes within the network. Alternatively, interconnectivity can be modeled in exemplar based theories with localist representations embedded in highly interconnected networks (Bybee, 2001; Hay \& Baayen, 2005). In both cases, the effect of word frequency need not be limited only to the word itself, but also affects its interconnections with other units in the network.

In both approaches, these are highly complex systems for which it may well be unrealistic to try to isolate the effect of one specific lexical measure. Our predictors offer but crude quantifications of aspects of these complex systems. None of our predictors take the complexity of the system into account in a principled way. The price we pay for the crudeness of our measures is rampant collinearity. The flip side is that this collinearity may itself be an index of the interconnectivity of the complex systems that we study. This interconnectivity may actually be fundamental to the robustness that characterizes lexical processing. From this perspective, the clustering of lexical measures that we have documented in the present study is the signature of the extensive covariation between the many formal and semantic properties that characterize the denizens of the mental lexicon. If this metaphor contains a grain of truth, then the role of morphological connectivity and of word frequency are intertwined to an even greater degree than we have heretofore envisioned.

\section{Acknowledgment}

This research was supported in part by Grant NIH HD-01994 to Haskins Laboratories. 


\section{References}

Allen, P., Wallace, B., \& Weber, T. (1995). Instance-based learning algorithms. Journal of Experimental Psychology: Human perception and performance, 21, 914-934.

Andrews, S. (1986). Morphological influences on lexical access: lexical or non-lexical effects? Journal of Memory and Language, 25, 726-740.

Andrews, S. (1989). Frequency and neighborhood size effects on lexical access: activation or search? Journal of Experimental Psychology: Learning, Memory, and Cognition, 15, 802-814.

Andrews, S. (1992). Frequency and neighborhood effects on lexical access: lexical similarity or orthographic redundancy? Journal of Experimental Psychology: Learning, Memory, \& Cognition, 18, 234-254.

Andrews, S. (1997). The effects of orthographic similarity on lexical retrieval: resolving neighborhood conflicts. Psychological Bulletin \& Review, 4, 439-461.

Baayen, R. (2005). Data mining at the intersection of psychology and linguistics. In A. Cutler (Ed.), Twenty-first century psycholinguistics: four cornerstones (pp. 69-83). Hillsdale, New Jersey: Erlbaum.

Baayen, R. H (2001). Word frequency distributions. Dordrecht: Kluwer Academic Publishers.

Baayen, R. H., \& Moscoso del Prado Martín, F. (2005). Semantic density and past-tense formation in three Germanic languages. Language, 81, 666-698.

Baayen, R. H, Piepenbrock, R., \& Gulikers, L. (1995). The CELEX lexical database (CD-ROM) Linguistic Data Consortium. Philadelphia, PA: Pennsylvania.

Balota, D., Cortese, M., \& Pilotti, M. (1999). Visual lexical decision latencies for 2906 words. [On-line], Available: $<$ http://www.artsci.wustl.edu/ dbalota/lexical_decision.html/>.

Balota, D., Cortese, M., Sergent-Marshall, S., Spieler, D., \& Yap, M. (2004). Visual word recognition for single-syllable words. Journal of Experimental Psychology: General, 133, 283-316.

Balota, D. A., \& Chumbley, J. I. (1984). Are lexical decisions a good measure of lexical access? The role of word frequency in the neglected decision stage. Journal of Experimental Psychology: Human Perception and Performance, 10, 340-357.

Balota, D. A., Pilotti, M., \& Cortese, M. J. (2001). Subjective frequency estimates ofr 2,938 monosyllabic words. Memory \& Cognition, 29, 639-647.

Barry, C., Hirsh, K., Johnston, R. A., \& Williams, C. (2001). Age of acquisition, word frequency, and the locus of repetition priming of picture naming. Journal of Memory and Language, 44, 350-375.

Bates, E., D'Amico, S., Jacobsen, T., Szekely, A., Andonova, E., Devescovi, A., et al. (2003). Timed picture naming in seven languages. Psychonomic Bulletin \& Review, 10, 344-380.

Beckwith, R., Fellbaum, C., Gross, D., \& Miller, G. (1991). WordNet: a lexical database organized on psycholinguistic principles. In U. Zernik (Ed.). Lexical Acquisition. Exploiting On-Line Resources to Build a Lexicon (pp. 211-232). Hillsdale, NJ: Lawrence Erlbaum Associates.

Belsley, D. A, Kuh, E., \& Welsch, R. E (1980). Regression Diagnostics. Identifying Influential Data and sources of
Collinearity. Wiley Series in Probability and Mathematical Statistics. New York: Wiley.

Bertram, R., Baayen, R. H., \& Schreuder, R. (2000). Effects of family size for complex words. Journal of Memory and Language, 42, 390-405.

Biber, D. (1988). Variation across speech and writing. Cambridge: Cambridge University Press.

Biber, D. (1995). Dimensions of register variation. Cambridge: Cambridge University Press.

Bird, H., Franklin, S., \& Howard, D. (2001). Age of acquisition and imageability ratings for a large set of words, including verbs and function words. Behavior Research Methods, Instruments, and Computers, 33, 73-79.

Brysbaert, M. (1996). Word frequency affects naming latency in Dutch with age-of-acquisition controlled. European Journal of Cognitive Psychology, 8, 185-193.

Brysbaert, M., Lange, M., \& Van Wijnendaele, I. (2000). The effects of age-of-acquisition and frequency-of-occurence in visual word recognition: further evidence from the Dutch language. European Journal of Cognitive Psychology, 12, 65-85.

Burnard, L. (1995). Users guide for the British National Corpus. British National Corpus consortium, Oxford university computing service.

Bybee, J. L (2001). Phonology and language use. Cambridge: Cambridge University Press.

Carroll, J. B (1967). On sampling from a lognormal model of word frequency distribution. In H. Kučera \& W. N. Francis (Eds.), Computational analysis of present-day American English (pp. 406-424). Providence: Brown University Press.

Carroll, J. B., \& White, M. N. (1973a). Age of acquisition norms for 220 picturable nouns. Journal of Verbal Learning and Verbal Behavior, 12, 563-576.

Carroll, J. B., \& White, M. N. (1973b). Word frequency and age of acquisition as determiners of picture-naming latency. Quarterly Journal of Experimental Psychology, 25, 85-95.

Chatterjee, S., Hadi, A. S., \& Price, B. (2000). Regression analysis by example. New York: John Wiley \& Sons.

Cohen, J., Cohen, P., West, S. G, \& Aiken, L. S (2003). Applied multiple regression/correlation analysis for the behavioral sciences. Hillsdale, New Jersey: Lawrence Erlbaum Associates.

Coltheart, M., Davelaar, E., Jonasson, J. T, \& Besner, D. (1977). Access to the internal lexicon. In S. Dornick (Ed.). Attention and performance (VI, pp. 535-556). Hillsdale, New Jersey: Erlbaum.

Cortese, M., \& Fugett, A. (2004). Imageability ratings for 3000 monosyllabic words. Behavioral methods and research, instrumentation, and computers, 36, 384-387.

De Jong, N. H. (2002). Morphological families in the mental lexicon. PhD thesis, University of Nijmegen, Nijmegen, The Netherlands.

De Jong, N. H., Schreuder, R., \& Baayen, R. H. (2003). Morphological resonance in the mental lexicon. In R. H. Baayen \& R. Schreuder (Eds.), Morphological structure in language processing (pp. 65-88). Berlin: Mouton de Gruyter.

Dijkstra, T., Moscoso del Prado Martín, F., Schulpen, B., Schreuder, R., \& Baayen, R. (2005). A roommate in cream: morphological family size effects on interlingual homograph recognition. Language and Cognitive Processes, 20, 7-41. 
Feldman, L., \& Pastizzo, M. (2003). Morphological facilitation: the role of semantic transparency and family size. In R. H. Baayen \& R. Schreuder (Eds.), Morphological structure in language processing (pp. 233-258). Berlin: Mouton de Gruyter.

Feldman, L. B. \& Basnight-Brown, D. (2005). The role of morphology in visual word recognition: Stem senses and semantic richness. In E. Grigorenko \& A. Naples (Eds.), Single word reading, (in press). Mahwah, NJ, Lawrence Erlbaum.

Fellbaum, C. e (1998). WordNet: an electronic database. Cambridge, MA: The MIT Press.

Forster, K., \& Shen, D. (1996). No enemies in the neighborhood: absence of inhibitory neighborhood effects in lexical decision and semantic categorization. Journal of Experimental Psychology: Learning, Memory, and Cognition, 22, 696-713.

Gernsbacher, M. A. (1984). Resolving 20 years of inconsistent interactions between lexical familiarity and orthography, concreteness, and polysemy. Journal of Experimental Psychology: General, 113, 256-281.

Grainger, J. (1990). Word frequency and neighborhood frequency effects in lexical decision and naming. Journal of Memory and Language, 29, 228-244.

Grainger, J. (1992). Orthographic neighborhoods and visual word recognition. In L. Katz \& R. Frost (Eds.), Orthography, Phonology, Morphology and Meaning (pp. 131-146). Amsterdam: Elsevier.

Grainger, J., \& Jacobs, A. M. (1996). Orthographic processing in visual word recognition: a multiple read-out model. Psychological Review, 103, 518-565.

Harm, M. W., \& Seidenberg, M. S. (2004). Computing the meanings of words in reading: cooperative division of labor between visual and phonological processes. Psychological Review, 111, 662-720.

Harrell, F. (2001). Regression modeling strategies. Berlin: Springer.

Hay, J. B., \& Baayen, R. H. (2005). Shifting paradigms: gradient structure in morphology. Trends in Cognitive Sciences, 9, 342-348.

Hocking, R. R (1996). Methods and applications of linear models. Regression and the analysis of variance. New York: Wiley.

Jastrzembski, J. (1981). Multiple meanings, number of related meanings, frequency of occurrence, and the lexicon. Cognitive Psychology, 13, 278-305.

Katz, L., \& Feldman, L. B. (1983). Relation between pronunciation and recognition of printed words in deep and shallow orthographies. Journal of Experimental Psychology: Learning, Memory, and Cognition, 9, 157-166.

Kostić, A. (1995). Informational load constraints on processing inflected morphology. In L. B. Feldman (Ed.), Morphological Aspects of Language Processing. New Jersey: Lawrence Erlbaum Inc. Publishers.

Kostić, A., Marković, T., \& Baucal, A. (2003). Inflectional morphology and word meaning: orthogonal or co-implicative domains? In R. H. Baayen \& R. Schreuder (Eds.), Morphological structure in language processing (pp. 1-44). Berlin: Mouton de Gruyter.

Kučera, H., \& Francis, W. N (1967). Computational Analysis of Present-Day American English. Providence, RI: Brown University Press.
Lupker, S. J. (1979). The semantic nature of response competition in the picture-word interference task. Memory \& Cognition, 7, 485-495.

MetaMetrics, I. (2003). MetaMetrics word frequency counts [database]. Author (Available from MetaMetrics Inc., developers of the Lexile Framework, attention: A. Jackson Stenner, 2327 Englert Drive, Suite 300, Durham NC 27713), Durham, NC.

Miller, G. A. (1990). Wordnet: an on-line lexical database. International Journal of Lexicography, 3, 235-312.

Monsell, S., Doyle, M. C., \& Haggard, P. N. (1989). Effects of frequency on visual word recognition tasks. Journal of Experimental Psychology: General, 118, 43-71.

Moscoso del Prado Martín, F., Bertram, R., Häikiö, T., Schreuder, R., \& Baayen, R. H. (2004). Morphological family size in a morphologically rich language: the case of finnish compared to Dutch and hebrew. Journal of Experimental Psychology: Learning, Memory, and Cognition, 30, 1271-1278.

Moscoso del Prado Martín, F., Deutsch, A., Frost, R., Schreuder, R., De Jong, N. H., \& Baayen, R. H. (2005). Changing places: a cross-language perspective on frequency and family size in Hebrew and Dutch. Journal of Memory and Language, 53, 496-512.

Moscoso del Prado Martín, F., Kostić, A., \& Bayen, R. H. (2004). Putting the bits together: an information theoretical perspective on morphological processing. Cognition, 94, 1-18.

Murray, W. S., \& Forster, K. (2004). Serial mechanisms in lexical access: the rank hypothesis. Psychological Review, $111,721-756$.

Nelson, D. L., McEvoy, C. L., \& Schreiber, T. A. (1998). The University of South Florida word association, rhyme, and word fragment norms. [On-line], Available: : $<$ http:// www.usf.eduFreeAssociation/>.

New, B., Ferrand, L., Pallier, C., \& Brysbaert, M. (in press). Re-examining word length effects in visual word recognition: new evidence from the English Lexicon Project. Psychonomic Bulletin \& Review.

R Development Core Team. (2005). R: A language and environment for statistical computing. R Foundation for Statistical Computing, Vienna, Austria. ISBN 3-900051-07-0.

Renouf, A. (1987). Corpus development. In J. M. Sinclair (Ed.), Looking up: A account of the Cobuild Project in Lexical Computing (pp. 1-40). London: Collins.

Rousseeuw, P., Struyf, A., \& Hubert, M. (2005). Cluster: functions for clustering. R port by Kurt Hornik (Kurt.Hornik@R-project.org) and Martin Maechler who has added several extensions. R package version 1.9.8.

Rubenstein, H., \& Pollack, I. (1963). Word predictability and intelligibility. Journal of Verbal Learning and Verbal Behavior, 2, 147-158.

Scarborough, D. L., Cortese, C., \& Scarborough, H. S. (1977). Frequency and repetition effects in lexical memory. Journal of Experimental Psychology: Human Perception and Performance, 3, 1-17.

Schreuder, R., \& Baayen, R. H. (1997). How complex simplex words can be. Journal of Memory and Language, 37, 118-139.

Seidenberg, M. S., \& Gonnerman, L. M. (2000). Explaining derivational morphology as the convergence of codes. Trends in Cognitive Sciences, 4, 353-361. 
Seidenberg, M. S., \& McClelland, J. L. (1989). A distributed, developmental model of word recognition and naming. Psychological Review, 96, 523-568.

Shapiro, B. J. (1969). The subjective estimation of word frequency. Journal of Verbal Learning and Verbal Behavior, 8, 248-251.

Spieler, D. H. \& Balota, D. A. (1998). Naming latencies for 2820 words. [On-line], Available from: <http://www. artsci.wustl.edu/ dbalota/naming.html/>.

Steyvers, M., \& Tanenbaum, J. (2005). Large-scale structure of semantic networks: statistical analyses and a model of semantic growth. Cognitive Science, 29, 41-78.

Tabak, W., Schreuder, R., \& Baayen, R. H (2005). Lexical statistics and lexical processing: semantic density, information complexity, sex, and irregularity in Dutch. In S. Kepser \& M. Reis (Eds.), Linguistic evidence-Empirical, Theoretical, and Computational Perspectives (pp. 529-555). Berlin: Mouton de Gruyter.
Toglia, M. P, \& Battig, W. (1978). Handbook of semantic word norms. Hillsdale, NJ: Erlbaum.

Traficante, D., \& Burani, C. (2003). Visual processing of Italian verbs and adjectives: the role of the inflectional family size. In R. H. Baayen \& R. Schreuder (Eds.), Morphological structure in language processing (pp. 45-64). Berlin: Mouton de Gruyter.

Venables, W. N, \& Ripley, B. (2003). Modern applied statistics with $R$. New York: Springer.

Wood, S. N (2006). Generalized additive models. An introduction with $R$. New York: Chapman \& Hall/CRC.

Zeno, S., Ivens, S., Millard, R., \& Duvvuri, R. (1995). The educator's word frequency guide. New York: Touchstone Applied Science.

Zevin, J., \& Seidenberg, M. (2002). Age of acquisition effects in word reading and other tasks. Journal of Memory and Language, 47, 1-29. 